\title{
A model problem concerning ionic transport in microstructured solid electrolytes
}

Received: 5 July 2014 / Accepted: 21 October 2014

(c) Springer-Verlag Berlin Heidelberg 2014

\begin{abstract}
We consider ionic transport by diffusion and migration through microstructured solid electrolytes. The assumed constitutive relations for the constituent phases follow from convex energy and dissipation potentials which guarantee thermodynamic consistency. The effective response is determined by homogenizing the relevant field equations via the notion of multi-scale convergence. The resulting homogenized response involves several effective tensors, but they all require the solution of just one standard conductivity problem over the representative volume element. A multi-scale model for semicrystalline polymer electrolytes with spherulitic morphologies is derived by applying the theory to a specific class of two-dimensional microgeometries for which the effective response can be computed exactly. An enriched model accounting for a random dispersion of filler particles with interphases is also derived. In both cases, explicit expressions for the effective material parameters are provided. The models are used to explore the effect of crystallinity and filler content on the overall response. Predictions support recent experimental observations on doped poly-ethylene-oxide systems which suggest that the anisotropic crystalline phase can actually support faster ion transport than the amorphous phase along certain directions dictated by the morphology of the polymeric chains. Predictions also support the viewpoint that ceramic fillers improve ionic conductivity and cation transport number via interphasial effects.
\end{abstract}

Keywords Diffusion · Migration · Heterogeneous solids · Periodic homogenization · Interphases

\section{Motivation}

Current efforts to develop polymeric electrolytes for all-solid-state lithium batteries are hampered by the unsatisfactory ionic conductivity and transport numbers of available polymers at room temperature. The most promising polymer electrolytes available to date consist of poly-ethylene-oxide (PEO) complexes doped with a lithium salt such as lithium perchlorate $\left(\mathrm{LiClO}_{4}\right)$ or lithium iodide (LiI). These are semicrystalline polymers often exhibiting spherulitic microstructures (e.g., [13,19,22]).

Early studies on different polymer complexes revealed that a transition from highly activated to easy ion transport with varying temperature was always accompanied by a considerable reduction in crystallinity [4]. This observation led to the widespread belief that only the amorphous phase could support

Communicated by Andreas Öchsner.

I. J. Curto Sillamoni · M. I. Idiart ( $\bigotimes)$

Departamento de Aeronáutica, Facultad de Ingeniería, Universidad Nacional de La Plata,

Avda. 1 esq. 47, B1900TAG La Plata, Argentina

E-mail: martin.idiart@ing.unlp.edu.ar

I. J. Curto Sillamoni · M. I. Idiart

CCT La Plata, Consejo Nacional de Investigaciones Científicas y Técnicas (CONICET), Calle 8 No 1467, B1904CMC La Plata, Argentina 
fast ion transport in semicrystalline polymers. Since then, efforts have focused on producing polymer complexes with minimal crystallinity levels - see [14] for a recent review. However, more recent studies have revealed that the ionic conductivity of PEO complexes can increase by several orders of magnitude when specimens are subjected to large stretchings $[16,17,21]$. This observation strongly suggests that the anisotropic crystalline phase can actually support faster ion transport than the amorphous phase along certain directions that become aligned with the principal axes of deformation. In fact, crystalline PEO is known to consist of monoclinic unit cells of aligned chains exhibiting a helical configuration which may permit fast ion transport along the helical axis (e.g., [15]). This suggests, in turn, that highly conductive PEO complexes could be obtained by tuning the crystallographic texture rather than reducing the crystallinity.

At the same time, studies on different composite polymer complexes revealed that ionic conductivity and cation transport number can increase upon the addition of a ceramic filler such as aluminum oxide $\left(\mathrm{Al}_{2} \mathrm{O}_{3}\right)$ or zirconium dioxide $\left(\mathrm{ZrO}_{2}\right)$ (e.g., $\left.[8,10,11]\right)$. Since ceramic fillers do not actually conduct ions, such increases have been ascribed to the presence of an amorphous interphase surrounding the filler particles that facilitates cation mobility while providing anchoring sites for anions. Further support to this belief stems from the fact that surface treatments to the filler particles can have a significant impact on the overall transport properties of the composite polymer (e.g., [10]).

The problem of correlating the overall ionic transport properties with the underlying microstructure in solid electrolytes is a difficult task requiring, in general, the solution of a set of coupled nonlinear electrodiffusion equations with highly oscillating coefficients. It is therefore common practice to introduce severe simplifications in the analysis-for instance, the assumption that ionic transport is Ohmic-which preclude the use of the resulting models to describe the above-mentioned phenomena. The purpose of the present work is to consider ionic transport in microstructured solids more rigorously through a continuum model problem with multiple microstructural length scales. We begin in Sect. 2 by specifying the physical problem and recalling the field equations commonly used to describe ionic transport in solids containing an arbitrary number of ion families. The assumed constitutive relations for the constituent phases follow from convex energy and dissipation potentials which guarantee thermodynamic consistency. The overall response of a microstructured solid is obtained in Sect. 3 by homogenizing the field equations via the notion of multi-scale convergence of [3]. The resulting homogenized response involves several effective tensors, but they all require the solution of just one standard conductivity problem over the representative volume element. The theory is applied in Sect. 4 to a model class of two-dimensional microgeometries which represent the essential morphological features of spherulitic polymer systems and at the same time allow exact computation of the overall response. An enriched model for composite systems consisting of a spherulitic polymer with a random dispersion of second-phase particles is also derived. In both cases, explicit expressions for the effective material parameters are provided. The models are used to explore the effect of crystallinity and filler content on the overall response of semicrystalline polymer electrolytes.

\section{Ionic transport in microstructured electrolytes}

We consider the transport of ions through a microstructured electrolyte medium composed of $N$ different homogeneous phases and operating under isothermal stationary conditions. We neglect any effects due to deformation. The electrolyte occupies a fixed domain $\Omega \subset \mathbb{R}^{3}$, while each phase $r$ occupies apossibly discontinuous-domain $\Omega^{(r)} \subset \Omega(r=1, \ldots, N)$ such that $\Omega=\cup_{r=1}^{N} \Omega^{(r)}$. The domains $\Omega^{(r)}$ can be described by a set of characteristic functions $\chi^{(r)}(\mathbf{x})$, which take the value 1 if the position vector $\mathbf{x}$ is in $\Omega^{(r)}$ and 0 otherwise. The phases contain $A$ different ionic species with valencies $z_{\alpha}$ $(\alpha=1, \ldots, A)$. In the presence of an electric field, these ions move through the electrolyte medium and conform an electric current. The focus here is on electrolyte systems where the length scales of the microstructural morphologies are much smaller than the characteristic size of the specimen and the scale of variation of the boundary conditions. Under these circumstances, the effective properties of the microstructured electrolyte are expected to be independent of the specific form of external actions on the system. The mathematical derivations of the next section are particularly simpler when the external action consists of a prescribed volumetric supply of ions within the electrolyte, which is independent of the microstructure. 
Thus, let $h_{\alpha}(\mathbf{x})$ denote a molar supply rate per unit volume of species $\alpha$ in $\Omega$; the balance of ions then requires that

$$
\nabla \cdot \mathbf{j}_{\alpha}=h_{\alpha} \quad \text { in } \Omega, \quad \alpha=1, \ldots, A,
$$

where $c_{\alpha}(\mathbf{x})$ and $\mathbf{j}_{\alpha}(\mathbf{x})$ denote, respectively, the molar concentration and molar flux of ions of type $\alpha$ within the electrolyte. We associate with each ionic species a continuous electrochemical potential field $\mu_{\alpha}(\mathbf{x})$ characterizing the energy flow due to species transport-see below. In turn, assuming magnetic effects are negligible, the electric potential $\phi(\mathbf{x})$ in all space is solution to the Maxwell's equations

$$
\nabla \cdot \mathbf{d}=F \sum_{\alpha=1}^{A} z_{\alpha} c_{\alpha}, \quad \mathbf{d}=\epsilon_{0} \mathbf{e}+\mathbf{p}, \quad \mathbf{e}=-\nabla \phi \quad \text { in } \mathbb{R}^{3},
$$

where $F$ is Faraday's constant, $\epsilon_{0}$ is the permittivity of vacuum, $\mathbf{d}(\mathbf{x})$ is the electric displacement, $\mathbf{e}(\mathbf{x})$ is the intensity of the electric field, $\mathbf{p}(\mathbf{x})$ is the electric polarization of the electrolyte medium, and $\phi(\mathbf{x})$ is continuous and such that $\phi \rightarrow 0$ as $|\mathbf{x}| \rightarrow \infty$. Finally, the electric current density $\mathbf{i}(\mathbf{x})$ flowing within the electrolyte is given in terms of the molar fluxes by

$$
\mathbf{i}=F \sum_{\alpha=1}^{A} z_{\alpha} \mathbf{j}_{\alpha} \text { in } \Omega .
$$

For simplicity, we assume that the conditions

$$
\mu_{\alpha}=0 \text { and } \phi=0
$$

hold on the boundary of $\Omega$. Note that the boundary condition on $\phi$ decouples the electric field distributions within $\Omega$ and outside of $\Omega$. Note also that, in view of the material heterogeneity, the above equations should be understood in the sense of distributions, with the fields $c_{\alpha}(\mathbf{x}), \mu_{\alpha}(\mathbf{x}), \phi(\mathbf{x})$, and $\mathbf{p}(\mathbf{x})$ supported in $\Omega$.

Equations (1)-(2) must be supplemented by suitable constitutive laws consistent with the principles of thermodynamics. ${ }^{1}$ These are obtained here by following the procedure of [9]. Thus, the rate of external working $\mathcal{F}$ due to the external supply of ions is given by

$$
\mathcal{F}=\sum_{\alpha=1}^{A} \int_{\Omega} \mu_{\alpha} h_{\alpha} \mathrm{d} V
$$

where the product $\mu_{\alpha} h_{\alpha}$ represents the chemical energy supply associated with each species $\alpha$ (see, for instance, [18]). On the other hand, the free energy $\mathcal{E}$ and rate of dissipation $\mathcal{D}$ of the electrochemical system are taken to be of the form

$$
\begin{aligned}
& \mathcal{E}=\int_{\Omega} W\left(\mathbf{x}, \mathbf{p}, c_{1}, \ldots, c_{A}\right) \mathrm{d} \mathbf{x}+\int_{\mathbb{R}^{3}} \frac{\epsilon_{0}}{2}|\mathbf{e}|^{2} \mathrm{~d} V, \\
& \mathcal{D}=\int_{\Omega} \sum_{\alpha=1}^{A} \frac{\partial U}{\partial\left(\nabla \mu_{\alpha}\right)}\left(\mathbf{x}, c_{1}, \ldots, c_{A}, \nabla \mu_{1}, \ldots, \nabla \mu_{A}\right) \cdot \nabla \mu_{\alpha} \mathrm{d} V .
\end{aligned}
$$

In (6), the first term represents the energy of the electrolyte, while the second term represents the energy of the electric field; in (7), the integrand represents the rate of energy per unit volume dissipated within the electrolyte due to ionic transport. Any dissipation due to electric polarizability is thus neglected. The energy density $W(\mathbf{x}, \cdot, \cdot)$ and dissipation potential $U\left(\mathbf{x}, c_{1}, \ldots, c_{A}, \cdot\right)$ are assumed to be convex. In addition, $U$ is assumed to be positive and to take the value 0 at $c_{\alpha}=0$ and $\nabla \mu_{\alpha}=\mathbf{0}$. These properties guarantee positivity of dissipation as required by the laws of thermodynamics.

\footnotetext{
${ }^{1}$ Initial conditions, on the other hand, will not be required in the subsequent analysis.
} 
Now, the rate of dissipation (7) must be equal to the difference between the rate of external working (5) and the rate of change of free energy (6) for all admissible thermodynamic processes. This implies that

$$
\int_{\Omega} \sum_{\alpha=1}^{A}\left(\mu_{\alpha}-\frac{\partial W}{\partial c_{\alpha}}-F z_{\alpha} \phi\right) \dot{c}_{\alpha} \mathrm{d} V+\int_{\Omega}\left(\mathbf{e}-\frac{\partial W}{\partial \mathbf{p}}\right) \cdot \dot{\mathbf{p}} \mathrm{d} V-\int_{\Omega} \sum_{\alpha=1}^{A}\left(\mathbf{j}_{\alpha}+\frac{\partial U}{\partial\left(\nabla \mu_{\alpha}\right)}\right) \cdot \nabla \mu_{\alpha} \mathrm{d} V=0
$$

must hold for all admissible thermodynamics processes—see, for instance, [26]. This, in turn, implies the constitutive relations

$$
\mathbf{e}=\frac{\partial W}{\partial \mathbf{p}}, \quad \mu_{\alpha}=\frac{\partial W}{\partial c_{\alpha}}+F z_{\alpha} \phi, \quad \mathbf{j}_{\alpha}=-\frac{\partial U}{\partial\left(\nabla \mu_{\alpha}\right)} .
$$

Further progress requires specific forms of the functions $W$ and $U$. These functions can be written as

$$
\begin{gathered}
W\left(\mathbf{x}, \mathbf{p}, c_{1}, \ldots, c_{A}\right)=\sum_{r=1}^{N} \chi^{(r)}(\mathbf{x}) W^{(r)}\left(\mathbf{p}, c_{1}, \ldots, c_{A}\right), \\
U\left(\mathbf{x}, c_{1}, \ldots, c_{A}, \nabla \mu_{1}, \ldots, \nabla \mu_{A}\right)=\sum_{r=1}^{N} \chi^{(r)}(\mathbf{x}) U^{(r)}\left(c_{1}, \ldots, c_{A}, \nabla \mu_{1}, \ldots, \nabla \mu_{A}\right),
\end{gathered}
$$

where the $W^{(r)}$ and $U^{(r)}$ denote the energy densities and dissipation potentials associated with each phase $r$. In this work, we characterize the electrochemical response of the constituent phases by functions of the form

$$
\begin{aligned}
W^{(r)}\left(\mathbf{p}, c_{1}, \ldots, c_{A}\right) & =\frac{1}{2} \mathbf{p} \cdot \boldsymbol{\kappa}^{(r)} \mathbf{p}+R T \sum_{\alpha=1}^{A} c_{\alpha}\left(\ln \frac{c_{\alpha}}{c_{0 \alpha}^{(r)}}-1\right), \\
U^{(r)}\left(c_{1}, \ldots, c_{A}, \nabla \mu_{1}, \ldots, \nabla \mu_{A}\right) & =\sum_{\alpha=1}^{A} \frac{1}{2} c_{\alpha} \nabla \mu_{\alpha} \cdot \mathbf{M}_{\alpha}^{(r)} \nabla \mu_{\alpha} .
\end{aligned}
$$

Here, $R T$ is the universal gas constant times the absolute temperature, $c_{0 \alpha}^{(r)}$ are reference molar concentrations, and $\boldsymbol{\kappa}^{(r)}$ and $\mathbf{M}_{\alpha}^{(r)}$ are positive-definite, second-order tensors characterizing, respectively, the electric polarizability and the ionic mobility in each phase $r$. Introducing these expressions in (9), we obtain the following constitutive relations consistent with thermodynamics:

$$
\mathbf{p}=\left(\kappa^{(r)}\right)^{-1} \mathbf{e}, \quad \mu_{\alpha}=R T \ln \frac{c_{\alpha}}{c_{0 \alpha}^{(r)}}+F z_{\alpha} \phi, \quad \mathbf{j}_{\alpha}=-c_{\alpha} \mathbf{M}_{\alpha}^{(r)} \nabla \mu_{\alpha} .
$$

These constitutive relations are known to be appropriate for low electric field intensities and dilute ion concentrations. Note that when the reference concentrations $c_{0}^{(r)}$ differ between phases, the molar concentrations $c_{\alpha}$ are discontinuous across material interfaces. In fact, the ratio $c_{0 \alpha}^{(r)} / c_{0 \alpha}^{(s)}$ fixes the ratio of molar concentrations of ionic species $\alpha$ in phases $r$ and $s$ under equilibrium conditions. Subsequent developments are facilitated by introducing the electric permittivity and pseudo-diffusivity tensors

$$
\boldsymbol{\epsilon}^{(r)}=\epsilon_{0} \mathbf{I}+\left(\boldsymbol{\kappa}^{(r)}\right)^{-1}, \quad \mathbb{D}_{\alpha}^{(r)}\left(\mu_{\alpha}, \phi\right)=g\left(\mu_{\alpha}-F z_{\alpha} \phi\right) c_{0 \alpha}^{(r)} \mathbf{M}_{\alpha}^{(r)},
$$

where

$$
g(s)=e^{s / R T}
$$

The exponential function in these expressions arises from elimination of the molar concentrations $c_{\alpha}$ by inversion of $(14)_{2}$. In view of these definitions, the constitutive relations for the molar fluxes and the electric displacement within each phase take the simple form

$$
\mathbf{j}_{\alpha}=-\mathbb{D}_{\alpha}^{(r)}\left(\mu_{\alpha}, \phi\right) \nabla \mu_{\alpha} \quad \text { and } \quad \mathbf{d}=\boldsymbol{\epsilon}^{(r)} \mathbf{e} .
$$

Note that the diffusivity of the various ion families are only coupled through the electric potential $\phi$. 


\section{The effective response}

The effective response of the microstructured electrolyte is formally obtained by evaluating the transport of ions through a sequence of material systems with fixed $\Omega$ and decreasingly smaller microstructural length scales. There are several ways of evaluating such a limit. In this work, we make use of the multi-scale convergence approach of [3]. This approach assumes the microstructure is a periodic repetition of cubic unit cells with possibly multiple length scales. Given our interest in semicrystalline polymer systems, we consider material systems possessing two well-separated microstructural length scales to be identified later with the characteristic sizes of the lamellae and the spherulites. More specifically, we let $\Omega_{\#}=[0, \ell]^{3}$ denote a cubic unit cell of side $\ell$ and write the characteristic functions $\chi^{(r)}$ as a product

$$
\chi^{(r)}(\mathbf{x})=\chi_{1}^{(r)}\left(\frac{\mathbf{x}}{\varepsilon}\right) \chi_{2}^{(r)}\left(\frac{\mathbf{x}}{\varepsilon^{2}}\right),
$$

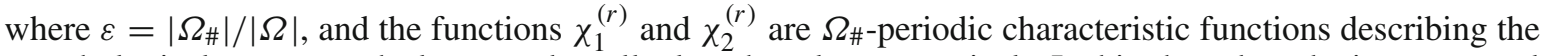
morphological patterns at the larger and smaller length scales, respectively. In this phase-based microstructural description, a given constituent phase may have to be labelled as two different materials in different mesoscale patterns; alternatively, multi-scale microstructures can be described by pattern-based characteristic functions as in [1].

In view of expressions (1) through (17), the corresponding sequences of electrochemical potentials $\mu_{\alpha}^{\varepsilon}$ and electric fields $\phi^{\varepsilon}$ in a stationary regime are solutions to the nonlinear field equations

$$
\begin{array}{cr}
-\nabla \cdot\left[\mathbb{D}_{\alpha}\left(\frac{\mathbf{x}}{\varepsilon}, \frac{\mathbf{x}}{\varepsilon^{2}}, \mu_{\alpha}^{\varepsilon}, \phi^{\varepsilon}\right) \nabla \mu_{\alpha}^{\varepsilon}\right]=h_{\alpha}(\mathbf{x}) & \text { in } \Omega, \\
-\nabla \cdot\left[\boldsymbol{\epsilon}\left(\frac{\mathbf{x}}{\varepsilon}, \frac{\mathbf{x}}{\varepsilon^{2}}\right) \nabla \phi^{\varepsilon}\right]=F \sum_{\alpha=1}^{A} z_{\alpha} c_{0 \alpha}\left(\frac{\mathbf{x}}{\varepsilon}, \frac{\mathbf{x}}{\varepsilon^{2}}\right) g\left(\mu_{\alpha}^{\varepsilon}-F z_{\alpha} \phi^{\varepsilon}\right) & \text { in } \Omega, \\
\mu_{\alpha}^{\varepsilon}=0 \text { and } \phi^{\varepsilon}=0 & \text { on } \partial \Omega,
\end{array}
$$

where

$$
\begin{aligned}
\mathbb{D}_{\alpha}\left(\mathbf{y}, \mathbf{z}, \mu_{\alpha}, \phi\right) & =\sum_{r=1}^{N} \chi_{1}^{(r)}(\mathbf{y}) \chi_{2}^{(r)}(\mathbf{z}) \mathbb{D}_{\alpha}^{(r)}\left(\mu_{\alpha}, \phi\right), \\
\boldsymbol{\epsilon}(\mathbf{y}, \mathbf{z}) & =\sum_{r=1}^{N} \chi_{1}^{(r)}(\mathbf{y}) \chi_{2}^{(r)}(\mathbf{z}) \boldsymbol{\epsilon}^{(r)} \\
c_{0 \alpha}(\mathbf{y}, \mathbf{z}) & =\sum_{r=1}^{N} \chi_{1}^{(r)}(\mathbf{y}) \chi_{2}^{(r)}(\mathbf{z}) c_{0 \alpha}^{(r)}
\end{aligned}
$$

$\alpha=1, \ldots, A$. The homogenized response of the system is obtained by taking the limit $\varepsilon \rightarrow 0$ in these equations.

Assuming the source terms $h_{\alpha}(\mathbf{x})$ are such that the sequences $\mu_{\alpha}^{\varepsilon}$ and $\phi^{\varepsilon}$ are bounded in $H_{0}^{1}(\Omega)$, these sequences multi-scale converge to some limit functions $\bar{\mu}_{\alpha}(\mathbf{x})$ and $\bar{\phi}(\mathbf{x})$ in $H_{0}^{1}(\Omega)$, and their gradients multiscale converge to some limit gradients $\nabla \bar{\mu}_{\alpha}(\mathbf{x})+\nabla_{y} \mu_{\alpha}^{1}(\mathbf{x}, \mathbf{y})+\nabla_{z} \mu_{\alpha}^{2}(\mathbf{x}, \mathbf{y}, \mathbf{z})$ and $\nabla \bar{\phi}(\mathbf{x})+\nabla_{y} \phi^{1}(\mathbf{x}, \mathbf{y})+$

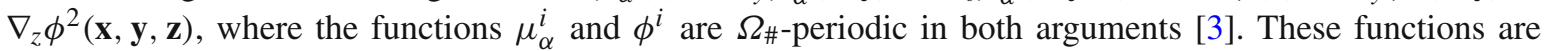
solution to the multi-scale equations

$$
\begin{aligned}
&-\nabla_{z} \cdot\left[\mathrm{M}_{\alpha}(\mathbf{y}, \mathbf{z})\left(\nabla \bar{\mu}_{\alpha}(\mathbf{x})+\nabla_{y} \mu_{\alpha}^{1}(\mathbf{x}, \mathbf{y})+\nabla_{z} \mu_{\alpha}^{2}(\mathbf{x}, \mathbf{y}, \mathbf{z})\right)\right]=0 \text { in } \Omega \times \Omega_{\#} \times \Omega_{\#}, \\
&-\nabla_{z} \cdot\left[\boldsymbol{\epsilon}(\mathbf{y}, \mathbf{z})\left(\nabla \bar{\phi}(\mathbf{x})+\nabla_{y} \phi^{1}(\mathbf{x}, \mathbf{y})+\nabla_{z} \phi^{2}(\mathbf{x}, \mathbf{y}, \mathbf{z})\right)\right]=0 \text { in } \Omega \times \Omega_{\#} \times \Omega_{\#}, \\
&-\nabla_{y} \cdot\left\langle\mathrm{M}_{\alpha}(\mathbf{y}, \mathbf{z})\left(\nabla \bar{\mu}_{\alpha}(\mathbf{x})+\nabla_{y} \mu_{\alpha}^{1}(\mathbf{x}, \mathbf{y})+\nabla_{z} \mu_{\alpha}^{2}(\mathbf{x}, \mathbf{y}, \mathbf{z})\right)\right\rangle_{z}=0 \text { in } \Omega \times \Omega_{\#}, \\
&-\nabla_{y} \cdot\left\langle\boldsymbol{\epsilon}(\mathbf{y}, \mathbf{z})\left(\nabla \bar{\phi}(\mathbf{x})+\nabla_{y} \phi^{1}(\mathbf{x}, \mathbf{y})+\nabla_{z} \phi^{2}(\mathbf{x}, \mathbf{y}, \mathbf{z})\right)\right\rangle_{z}=0 \text { in } \Omega \times \Omega_{\#}, \\
&-\nabla \cdot\left\langle\mathbb{D}_{\alpha}\left(\mathbf{y}, \mathbf{z}, \bar{\mu}_{\alpha}, \bar{\phi}\right)\left(\nabla \bar{\mu}_{\alpha}(\mathbf{x})+\nabla_{y} \mu_{\alpha}^{1}(\mathbf{x}, \mathbf{y})+\nabla_{z} \mu_{\alpha}^{2}(\mathbf{x}, \mathbf{y}, \mathbf{z})\right)\right\rangle_{y, z}=h_{\alpha}(\mathbf{x}) \quad \text { in } \Omega,
\end{aligned}
$$




$$
\begin{gathered}
-\nabla \cdot\left\langle\boldsymbol{\epsilon}(\mathbf{y}, \mathbf{z})\left(\nabla \bar{\phi}(\mathbf{x})+\nabla_{y} \phi^{1}(\mathbf{x}, \mathbf{y})+\nabla_{z} \phi^{2}(\mathbf{x}, \mathbf{y}, \mathbf{z})\right)\right\rangle_{y, z}=F \sum_{\alpha=1}^{A} z_{\alpha}\left\langle c_{0 \alpha}\right\rangle_{y, z} g\left(\bar{\mu}_{\alpha}-F z_{\alpha} \bar{\phi}\right) \\
\text { in } \Omega, \\
\bar{\mu}_{\alpha}=0 \text { and } \bar{\phi}=0 \text { on } \partial \Omega,
\end{gathered}
$$

$\alpha=1, \ldots, A$. In these expressions, $\nabla_{y}$ and $\nabla_{z}$ refer to the partial nabla operator with respect to $\mathbf{y}$ and $\mathbf{z}$, respectively, $\langle\cdot\rangle_{y},\langle\cdot\rangle_{z}$, and $\langle\cdot\rangle_{y, z}$ denote volume averages over $\mathbf{y}$, over $\mathbf{z}$, and over both $\mathbf{y}$ and $\mathbf{z}$, respectively, and

$$
\mathbb{M}_{\alpha}(\mathbf{y}, \mathbf{z})=\sum_{r=1}^{N} \chi_{1}^{(r)}(\mathbf{y}) \chi_{2}^{(r)}(\mathbf{z}) \mathbb{M}_{\alpha}^{(r)} \quad \text { with } \quad \mathbb{M}_{\alpha}^{(r)}=\frac{c_{0 \alpha}^{(r)}}{\left\langle c_{0 \alpha}\right\rangle_{y, z}} \mathbf{M}_{\alpha}^{(r)} .
$$

Equations (25), (27), and (29) follow from the multi-scale limit of Eq. (19), while Eqs. (26), (28), and (30) follow from the multi-scale limit of Eq. (20). The proofs are given in Appendix 1. The functions $\bar{\mu}_{\alpha}(\mathbf{x})$ and $\bar{\phi}(\mathbf{x})$ represent the macroscopic electrochemical and electric potentials, while the functions $\mu_{\alpha}^{i}$ and $\phi^{i}$ are related to the underlying microscopic potentials. Note that the microscopic/mesoscopic Eqs. (25)-(28) are linear, and it is only the macroscopic Eqs. (29)-(30) that preserve the nonlinearity of the original field equations. In fact, in the homogenization limit $(\varepsilon \rightarrow 0)$, the microscopic electrochemical and electric potentials are coupled only via the macroscopic gradients. In other words, for a given macroscopic electric field, the microscopic electric potential is independent of the electrochemical potentials; and for a given macroscopic gradient of an electrochemical potential, the corresponding microscopic electrochemical potential is independent of the other electrochemical potentials and the electric potential. This is a consequence of the separation of length scales and the assumed additive decomposition of the functions (12) and (13). Note also that the ion mobility at the microscopic scale is dictated by the weighted mobility tensors (32).

The form of Eqs. (25)-(31) motivate the definition of the following microscopic, mesoscopic ( $)$ ), and macroscopic (·) quantities:

$$
\begin{aligned}
\mathbf{e}(\mathbf{x}, \mathbf{y}, \mathbf{z}) & =-\left[\nabla \bar{\phi}(\mathbf{x})+\nabla_{y} \phi^{1}(\mathbf{x}, \mathbf{y})+\nabla_{z} \phi^{2}(\mathbf{x}, \mathbf{y}, \mathbf{z})\right], \\
\widetilde{\mathbf{e}}(\mathbf{x}, \mathbf{y}) & =\langle\mathbf{e}(\mathbf{x}, \mathbf{y}, \mathbf{z})\rangle_{z}=-\left[\nabla \bar{\phi}(\mathbf{x})+\nabla_{y} \phi^{1}(\mathbf{x}, \mathbf{y})\right], \\
\overline{\mathbf{e}}(\mathbf{x}) & =\langle\widetilde{\mathbf{e}}(\mathbf{x}, \mathbf{y})\rangle_{y}=-\nabla \bar{\phi}(\mathbf{x}), \\
\mathbf{d}(\mathbf{x}, \mathbf{y}, \mathbf{z}) & =\boldsymbol{\epsilon}(\mathbf{y}, \mathbf{z}) \mathbf{e}(\mathbf{x}, \mathbf{y}, \mathbf{z}), \\
\widetilde{\mathbf{d}}(\mathbf{x}, \mathbf{y}) & =\langle\mathbf{d}(\mathbf{x}, \mathbf{y}, \mathbf{z})\rangle_{z}=\widetilde{\boldsymbol{\epsilon}}(\mathbf{y}) \widetilde{\mathbf{e}}(\mathbf{x}, \mathbf{y}), \\
\overline{\mathbf{d}}(\mathbf{x}) & =\langle\widetilde{\mathbf{d}}(\mathbf{x}, \mathbf{y})\rangle_{y}=\overline{\boldsymbol{\epsilon}} \overline{\mathbf{e}}(\mathbf{x}), \\
\mathbf{j}_{\alpha}(\mathbf{x}, \mathbf{y}, \mathbf{z}) & =-g\left(\bar{\mu}_{\alpha}-F z_{\alpha} \bar{\phi}\right)\left\langle c_{0 \alpha}\right\rangle_{y, z} \mathbb{M}_{\alpha}(\mathbf{y}, \mathbf{z})\left[\nabla \bar{\mu}_{\alpha}(\mathbf{x})+\nabla_{y} \mu_{\alpha}^{1}(\mathbf{x}, \mathbf{y})+\nabla_{z} \mu_{\alpha}^{2}(\mathbf{x}, \mathbf{y}, \mathbf{z})\right], \\
\widetilde{\mathbf{j}} \alpha(\mathbf{x}, \mathbf{y}) & =\left\langle\mathbf{j}_{\alpha}(\mathbf{x}, \mathbf{y}, \mathbf{z})\right\rangle_{z}=-g\left(\bar{\mu}_{\alpha}-F z_{\alpha} \bar{\phi}\right)\left\langle c_{0 \alpha}\right\rangle_{y, z} \widetilde{\mathbb{M}}_{\alpha}(\mathbf{y})\left[\nabla \bar{\mu}_{\alpha}(\mathbf{x})+\nabla_{y} \mu_{\alpha}^{1}(\mathbf{x}, \mathbf{y})\right], \\
\overline{\mathbf{j}}_{\alpha}(\mathbf{x}) & =\left\langle\widetilde{\mathbf{j}}_{\alpha}(\mathbf{x}, \mathbf{y})\right\rangle_{y}=-g\left(\bar{\mu}_{\alpha}-F z_{\alpha} \bar{\phi}\right)\left\langle c_{0 \alpha}\right\rangle_{y, z} \overline{\mathbb{M}}_{\alpha} \nabla \bar{\mu}_{\alpha}(\mathbf{x})=-\overline{\mathbb{D}}_{\alpha}\left(\bar{\mu}_{\alpha}, \bar{\phi}\right) \nabla \bar{\mu}_{\alpha}(\mathbf{x}), \\
\bar{c}_{\alpha}(\mathbf{x}) & =\left\langle c_{0 \alpha}\right\rangle_{y, z} g\left(\bar{\mu}_{\alpha}-F z_{\alpha} \bar{\phi}\right) .
\end{aligned}
$$

The second identities in (37)-(41) follow from the linear dependence of the local fields $\mu_{\alpha}^{i}$ and $\phi^{i}$ on the macroscopic gradients $\nabla \bar{\mu}_{\alpha}$ and $\nabla \bar{\phi}$. In fact, the local problems (25) through (28) have the structure of a standard linear conductivity problem. Thus, the effective tensors at the mesoscopic level can be expressed as

$$
\widetilde{\mathbb{M}}_{\alpha}(\mathbf{y})=\left\langle\mathbb{M}_{\alpha}(\mathbf{y}, \mathbf{z}) \mathbf{A}_{2}\left\{\mathbb{M}_{\alpha}(\mathbf{y}, \mathbf{z})\right\}\right\rangle_{z} \text { and } \tilde{\boldsymbol{\epsilon}}(\mathbf{y})=\left\langle\boldsymbol{\epsilon}(\mathbf{y}, \mathbf{z}) \mathbf{A}_{2}\{\boldsymbol{\epsilon}(\mathbf{y}, \mathbf{z})\}\right\rangle_{z}
$$

while the effective tensors at the macroscopic level can be expressed as

$$
\overline{\mathrm{M}}_{\alpha}=\left\langle\widetilde{\mathbb{M}}_{\alpha}(\mathbf{y}) \mathbf{A}_{1}\left\{\widetilde{\mathbb{M}}_{\alpha}(\mathbf{y})\right\}\right\rangle_{y} \quad \text { and } \overline{\boldsymbol{\epsilon}}=\left\langle\widetilde{\boldsymbol{\epsilon}}(\mathbf{y}) \mathbf{A}_{1}\{\widetilde{\boldsymbol{\epsilon}}(\mathbf{y})\}\right\rangle_{y} .
$$


In these expressions, the tensors $\mathbf{A}_{i}\{\cdot\}$ are the standard concentration tensors of the gradient fields associated with the unit cell problems (25)-(28) (see, for instance, [23]). The tensors $\overline{\boldsymbol{\epsilon}}$ and $\overline{\mathrm{M}}_{\alpha}$ denote, respectively, the effective permittivity of the electrolyte and the effective mobilities of each ionic species $\alpha$ within the homogenized electrolyte. Note that at a given length scale (mesoscopic/macroscopic), all effective tensors are given in terms of the same set of concentration tensors $\mathbf{A}_{i}$ evaluated at different local constitutive tensors. Thus, the homogenized properties at a given length scale can be obtained by solving just one standard linear conductivity problem over the unit cell. A similar result has been recently obtained in a simpler context by [5] via two-scale asymptotic expansions. Motivated by a dimensional analysis of the various physical magnitudes involved, [5] also considered an alternative set of microscopic equations where the left-hand side of Gauss' equation (26) is multiplied by the small parameter $\varepsilon^{3}$, and obtained an alternative set of macroscopic equations with Gauss' equation replaced by an electroneutrality condition. A similar analysis could be performed by means of multi-scale convergence but is not pursued here for brevity.

With definitions (33)-(42), the multi-scale system of Eqs. (25)-(30) can be written as

$$
\begin{aligned}
\nabla_{z} \cdot \mathbf{j}_{\alpha}(\mathbf{x}, \mathbf{y}, \mathbf{z})=0, & \nabla_{z} \cdot \mathbf{d}(\mathbf{x}, \mathbf{y}, \mathbf{z})=0 & \text { in } \Omega \times \Omega_{\#} \times \Omega_{\#}, \\
\nabla_{y} \cdot \widetilde{\mathbf{j}}_{\alpha}(\mathbf{x}, \mathbf{y})=0, & \nabla_{y} \cdot \widetilde{\mathbf{d}}(\mathbf{x}, \mathbf{y})=0 & \text { in } \Omega \times \Omega_{\#}, \\
\nabla_{x} \cdot \overline{\mathbf{j}}_{\alpha}(\mathbf{x})=h_{\alpha}(\mathbf{x}), & \nabla_{x} \cdot \overline{\mathbf{d}}(\mathbf{x})=F \sum_{\alpha=1}^{A} z_{\alpha} \bar{c}_{\alpha}(\mathbf{x}) & \text { in } \Omega .
\end{aligned}
$$

Finally, we can define microscopic, mesoscopic, and macroscopic current densities as

$$
\begin{aligned}
\mathbf{i}(\mathbf{x}, \mathbf{y}, \mathbf{z}) & =F \sum_{\alpha=1}^{A} z_{\alpha} \mathbf{j}_{\alpha}(\mathbf{x}, \mathbf{y}, \mathbf{z}), \\
\widetilde{\mathbf{i}}(\mathbf{x}, \mathbf{y}) & =\langle\mathbf{i}(\mathbf{x}, \mathbf{y}, \mathbf{z})\rangle_{z}=F \sum_{\alpha=1}^{A} z_{\alpha} \widetilde{\mathbf{j}}_{\alpha}(\mathbf{x}, \mathbf{y}), \\
\overline{\mathbf{i}}(\mathbf{x}) & =\widetilde{\mathbf{i}}(\mathbf{x}, \mathbf{y})\rangle_{y}=F \sum_{\alpha=1}^{A} z_{\alpha} \overline{\mathbf{j}}_{\alpha}(\mathbf{x}) .
\end{aligned}
$$

Then, noting that the macroscopic molar concentrations $\bar{c}_{\alpha}$ given by (42) are continuous-unlike the microscopic molar concentrations $c_{\alpha}$-, we have that

$$
\overline{\mathbf{j}}_{\alpha}=-R T \sum_{\alpha=1}^{A} \overline{\mathrm{M}}_{\alpha} \nabla \bar{c}_{\alpha}+F \sum_{\alpha=1}^{A} z_{\alpha} \bar{c}_{\alpha} \overline{\mathrm{M}}_{\alpha} \overline{\mathbf{e}}
$$

and therefore

$$
\overline{\mathbf{i}}=-R T F \sum_{\alpha=1}^{A} z_{\alpha} \overline{\mathrm{M}}_{\alpha} \nabla \bar{c}_{\alpha}+F^{2} \sum_{\alpha=1}^{A} z_{\alpha}^{2} \bar{c}_{\alpha} \overline{\mathrm{M}}_{\alpha} \overline{\mathbf{e}},
$$

where use has been made of (41). In view of these expressions, and following common practice, we define the macroscopic ionic transport tensors

$$
\overline{\boldsymbol{D}}_{\alpha}=R T \overline{\mathrm{M}}_{\alpha} \text { and } \overline{\boldsymbol{\kappa}}=F^{2} \sum_{\alpha=1}^{A} z_{\alpha}^{2} \bar{c}_{\alpha} \overline{\mathrm{M}}_{\alpha},
$$

which represent the effective diffusivities and the effective conductivity of the microstructured electrolyte, respectively. It is pointed out that the conductivity depends on the ion concentrations $\bar{c}_{\alpha}$, and therefore, it is not a material parameter. 


\section{A multi-scale model for semicrystalline polymer electrolytes}

\subsection{Assumptions and formulae}

The focus here is on semicrystalline polymer electrolytes exhibiting spherulitic microstructures operating at room temperature. For definiteness, we take the PEO systems doped with $\mathrm{LiClO}_{4}$ of [13] as a case study. In these systems, the $\mathrm{PEO}$ molecules dissolve the $\mathrm{LiClO}_{4}$ salt into $\mathrm{Li}^{+}(\alpha=+)$ and $\mathrm{ClO}_{4}^{-}(\alpha=-)$ univalent ions $\left(z_{+}=\right.$ $\left.-z_{-}=1\right)$ and form various phases depending on salt concentration and temperature. At room temperature, their microstructural morphology is a two-scale structure formed by alternating layers of crystalline and amorphous phases in intimate contact with each other at the lower nanometer scale, which in turn radiate out from various center points in the specimen, leading to morphological structures at the larger micrometer scale known as spherulites. Depending on salt concentration, the spherulites can either form a granular aggregate or be separated by a continuous amorphous matrix, and the volume fraction of the crystalline phase can range from 10 to $90 \%$. At the molecular scale, the crystalline phase consists of monoclinic unit cells of aligned polymer chains exhibiting a helical configuration [15].

We derive a multi-scale model for these material systems by constructing (two-dimensional) microgeometries which, on the one hand, are complex enough to reproduce the essential geometrical features of the above microstructures, but, on the other hand, are simple enough so that their homogenized response can be computed exactly. The microgeometries of choice consist of a combination of a Schulgasser's cylinder assemblage (Schulgasser 1983) and simple laminates. At the mesoscale, these microgeometries are assemblages of homothetic cylinders of infinite sizes filling up the entire unit cell, each composed of an isotropic exterior coating of amorphous phase and a radially symmetric interior core or cylindrite. At the microscale, the cylindrite is in turn a laminated microgeometry composed of alternating laminae of amorphous and crystalline phases with the lamination direction coincident with the mesoscale tangential direction. A schematic of the microgeometries is shown in Fig. 1.

The microstructural parameters of the model are the volume fractions of the cylinders occupied by the amorphous rings $f_{r}$ and by the cylindrites $f_{c}$, and the volume fractions of the lamellar structure within the cylindrite occupied by the amorphous phase $f_{c}^{a}$ and by the crystalline phase $f_{c}^{\mathrm{cr}}$. These parameters satisfy the identities

$$
f_{r}+f_{c}=1 \text { and } f_{c}^{a}+f_{c}^{\mathrm{cr}}=1 .
$$

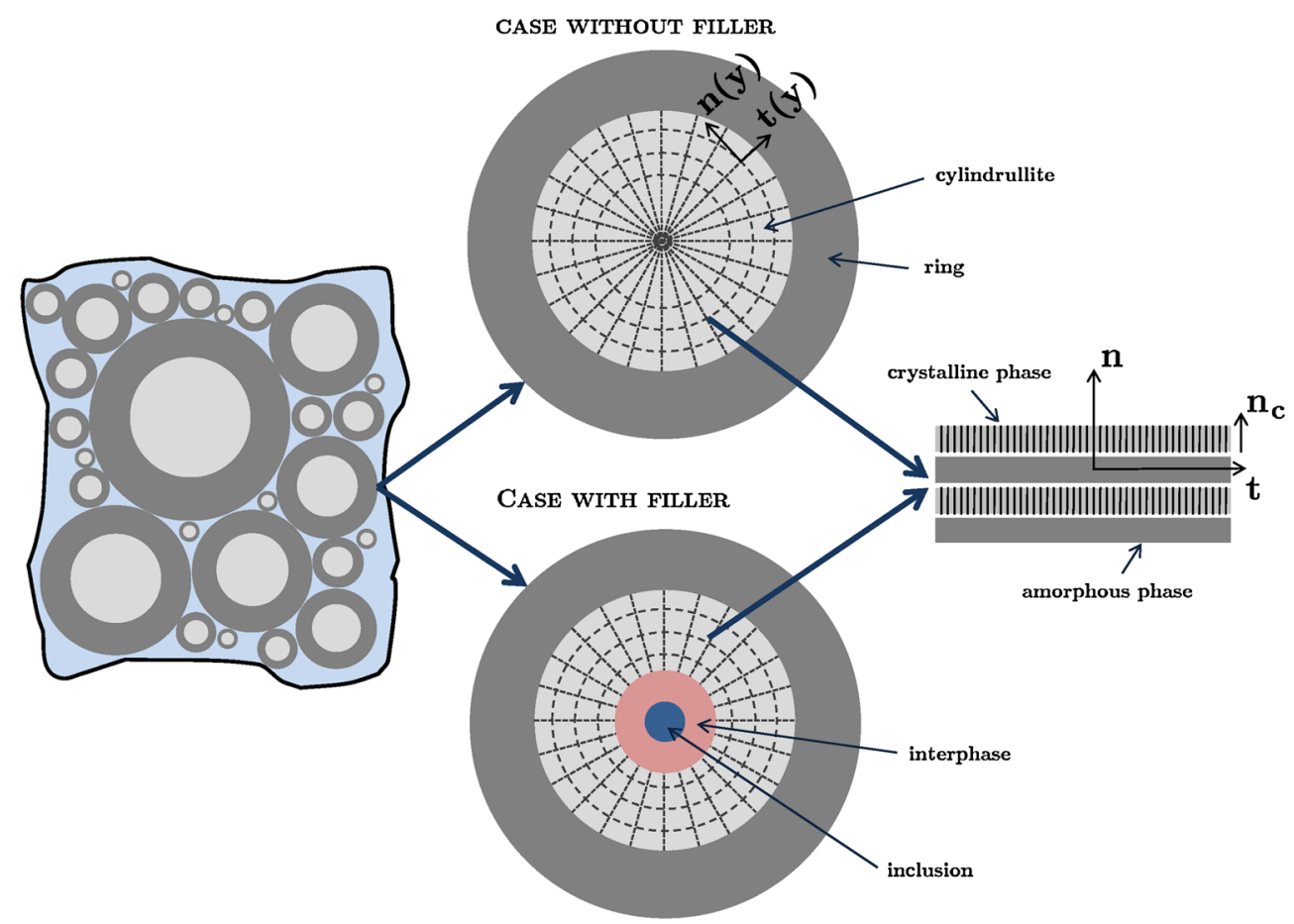

Fig. 1 Schematic of the Schulgasser's cylinder assemblages utilized in the models with and without filler inclusions 
The total volume fractions of crystalline and amorphous phases are thus given by

$$
f^{(\mathrm{cr})}=f_{c} f_{c}^{\mathrm{cr}} \text { and } f^{(a)}=f_{c} f_{c}^{a}+f_{r},
$$

respectively, and are such that $f^{(\mathrm{cr})}+f^{(a)}=1$; the volume fraction $f^{(\mathrm{cr})} \equiv f$ represents the crystallinity of the electrolyte.

The mobility tensors of both ion types in the isotropic amorphous phase and the anisotropic crystalline phase are taken to be, respectively,

$$
\mathbf{M}_{\alpha}^{(a)}=m_{\alpha}^{(a)} \mathbf{I} \quad \text { and } \quad \mathbf{M}_{\alpha}^{(\mathrm{cr})}=m_{\alpha}^{\|} \mathbf{n}_{c} \otimes \mathbf{n}_{c}+m_{\alpha}^{\perp}\left(\mathbf{I}-\mathbf{n}_{c} \otimes \mathbf{n}_{c}\right)
$$

where $m_{\alpha}^{\|}$and $m_{\alpha}^{\perp}$ represent mobilities along the directions parallel and perpendicular to the direction $\mathbf{n}_{c}$ of the chain helix axes. The corresponding weighted mobility tensors $\mathbb{M}_{\alpha}$ are given by

$$
\mathrm{I}_{\alpha}^{(a)}=\not h_{\alpha}^{(a)} \mathbf{I} \text { and } \quad \mathbb{M}_{\alpha}^{(\mathrm{cr})}=\not h_{\alpha}^{\|} \mathbf{n}_{c} \otimes \mathbf{n}_{c}+\not h_{\alpha}^{\perp}\left(\mathbf{I}-\mathbf{n}_{c} \otimes \mathbf{n}_{c}\right)
$$

with

$$
m_{\alpha}^{(a)}=\frac{c_{0 \alpha}^{(a)}}{f^{(\mathrm{cr})} c_{0 \alpha}^{(\mathrm{cr})}+f^{(a)} c_{0 \alpha}^{(a)}} m_{\alpha}^{(a)} \quad \text { and } \quad m_{\alpha}^{\|, \perp}=\frac{c_{0 \alpha}^{(\mathrm{cr})}}{f^{(\mathrm{cr})} c_{0 \alpha}^{(\mathrm{cr})}+f^{(a)} c_{0 \alpha}^{(a)}} m_{\alpha}^{\|, \perp} .
$$

Here, $c_{0 \alpha}^{(\mathrm{cr})}$ and $c_{0 \alpha}^{(a)}$ are the reference concentrations of the crystalline and amorphous phases, respectively.

Following the scheme of the previous section, the overall response is obtained sequentially by first homogenizing the laminated structure to obtain the mesoscopic mobilities $\widetilde{\mathrm{M}}_{\alpha}$ as dictated by $(43)_{1}$, and then homogenizing Schulgasser's assemblage to obtain the macroscopic mobilities $\overline{\mathrm{M}}_{\alpha}$ as dictated by (44) $)_{1}$. In so doing, it should be pointed out that, strictly, the multi-scale microgeometries considered here lie outside the class of periodic microgeometries considered in the previous section, where it is required that the unit cells at both scales be cubic and mutually aligned. However, it is reasonable to assume that the multi-scale Eqs. (33)-(42) remain valid more generally.

The ensuing analysis is greatly simplified by assuming that the chain axes $\mathbf{n}_{c}$ are aligned with the lamination direction $\mathbf{n}$. This amounts to disregarding chain tilting. Granted this assumption, the effective mobilities in the lamellae structure can be written as—-see, for instance, [20]—

$$
\widetilde{\mathrm{M}}_{\alpha}(\mathbf{y})=\widetilde{\not h}_{\alpha}^{\|} \mathbf{n}(\mathbf{y}) \otimes \mathbf{n}(\mathbf{y})+\widetilde{\not}_{\alpha}^{\perp}[\mathbf{I}-\mathbf{n}(\mathbf{y}) \otimes \mathbf{n}(\mathbf{y})],
$$

where $\mathbf{n}(\mathbf{y})$ is lamination direction at position $\mathbf{y}$ in the Schulgasser assemblage, and the parallel and perpendicular mobilities are given by

$$
\widetilde{m}_{\alpha}^{\|}=\left(\frac{f_{c}^{c r}}{\not \eta_{\alpha}^{\|}}+\frac{f_{c}^{a}}{m_{\alpha}^{(a)}}\right)^{-1} \text { and } \widetilde{\eta}_{\alpha}^{\perp}=f_{c}^{\mathrm{cr}} m_{\alpha}^{\perp}+f_{c}^{a} m_{\alpha}^{(a)} .
$$

Thus, the axes of anisotropy of the mesoscopic mobility tensors are radially aligned within the cylindrites. This allows the use of Schulgasser's solution scheme to obtain the macroscopic mobility tensors $\overline{\mathrm{M}}_{\alpha}$, which yields

$$
\overline{\mathrm{M}}_{\alpha}=\overline{\eta h}_{\alpha} \mathbf{I}
$$

with

$$
\bar{\eta}_{\alpha}=h_{\alpha}^{(a)} \frac{\left(1-f_{c}\right) \eta_{\alpha}^{(a)}+\left(1+f_{c}\right) \sqrt{\widetilde{m}_{\alpha}^{\|} \widetilde{m}_{\alpha}^{\perp}}}{\left(1+f_{c}\right) \eta_{\alpha}^{(a)}+\left(1-f_{c}\right) \sqrt{{\widetilde{\eta_{\alpha}}}_{\alpha}^{\|} \widetilde{m}_{\alpha}^{\perp}}} .
$$

Details of the derivation of this expression are given in Appendix 2. Expression (62), together with (58) and (60), constitutes a closed-form estimate for the mobilities of each ion species through the model semicrystalline 
polymer in terms of the local mobilities of the amorphous and crystalline phases, and the various microstructural parameters. In the absence of amorphous rings $\left(f_{c}=1\right)$, the expression reduces to

$$
\overline{\eta ্}_{\alpha}=\sqrt{\widetilde{m}_{\alpha}^{\|} \widetilde{m}_{\alpha}^{\perp}} \text {. }
$$

If, in turn, no amorphous phase is present in the lamellar structure $\left(f_{c}=f_{c}^{\mathrm{cr}}=f^{(\mathrm{cr})}=1\right)$, the expression reduces further to

$$
\overline{\eta n}_{\alpha}=\sqrt{m_{\alpha}^{\|} m_{\alpha}^{\perp}}
$$

Note that in all cases, $\bar{\eta}_{\alpha}$ is symmetric in $m_{\alpha}^{\|}$and $m_{\alpha}^{\perp}$ : Interchanging the parallel and perpendicular mobilities in the crystalline phase results in the same effective mobility.

Given the overall isotropy of the electrolyte, the effective conductivity is of the form $\bar{\kappa}=\bar{\kappa} \mathbf{I}$. In a typical lithium battery, it is only the cations that react at the electrode-electrolyte interfaces. Thus, under steady state conditions, there is no net flux of anions through the electrolyte and ionic conduction is solely due to cation transport. Consequently, we set

$$
\bar{\kappa}=F^{2} \bar{c}_{+} \bar{\eta}_{+} \cdot
$$

For later purposes, it is convenient to introduce a reference molar concentration $c_{0}=\rho_{\mathrm{PEO}} / w_{\mathrm{PEO}}$, where

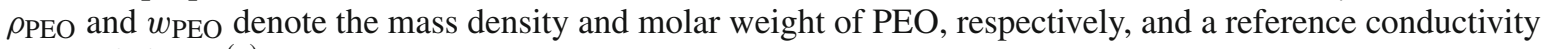
$\kappa_{0}=F^{2} z_{+}^{2} c_{0} m_{+}^{(a)}$, so that

$$
\frac{\bar{\kappa}}{\kappa_{0}}=\frac{\bar{c}_{+}}{c_{0}} \frac{\bar{m}_{+}}{m_{+}^{(a)}} .
$$

Finally, it is noted that if the electric permittivity tensors of the amorphous and crystalline phases are taken to be of the same form (56), the effective permittivity tensor $\overline{\boldsymbol{\epsilon}}$ of the electrolyte is given by the same expressions (61)-(62) with the weighted mobilities replaced by the local electric permittivities.

\subsection{The effect of crystallinity and salt concentration}

Here, we present a selected set of numerical results to explore the dependence of the macroscopic mobilities on the various material and microstructural parameters.

We begin by considering material systems with no amorphous ring $\left(f_{r}=0\right)$. The results are parameterized by the reference concentration contrast $c_{0 \alpha}^{\text {(cr) }} / c_{0 \alpha}^{(a)}$, the mobility contrast $\left(m_{\alpha}^{\|}+m_{\alpha}^{\perp}\right) /\left(2 m_{\alpha}^{(a)}\right)$, and the mobility anisotropy ratio of the crystalline phase $m_{\alpha}^{\|} / m_{\alpha}^{\perp}$. We assume that the mobilities are such that $m_{\alpha}^{\perp} \leq m_{\alpha}^{a} \leq m_{\alpha}^{\|}$. Moreover, given that the ratio of PEO ether oxygens to lithium ions in the crystalline phase is typically lower than in the amorphous phase, we assume that $c_{0 \alpha}^{(\mathrm{cr})} / c_{0 \alpha}^{(a)} \geq 1$. Figure 2 shows predictions for the effective mobility $\bar{m}_{\alpha}$ of species $\alpha$, normalized by the corresponding mobility in the amorphous phase $m_{\alpha}^{(a)}$, as a function of crystallinity $f$, for a wide range of material parameters. It is recalled that these normalized results are the same for both ionic species $\alpha$. The main observation in the context of this figure is that the effective mobility can decrease with increasing crystallinity even when the mobility in the crystalline phase along the chain axis is orders of magnitude higher than the mobility of the amorphous phase. In fact, the predictions provided in parts (a) and (b) show that for sufficiently low mobility contrasts $(\leq 5)$ and large mobility anisotropy ratios $(\geq 1,000)$, the effective mobility decreases monotonically with crystallinity, by up to an order of magnitude, in the range $0 \leq f \leq 0.9$. This shows that the decrease in ionic conductivity with crystallinity commonly observed in semicrystalline polymer electrolytes does not imply a faster ionic transport in the amorphous phase than in the crystalline phase, as originally proposed by [4]. Moreover, while this model does not account for the effect of mechanical deformations, it is clear that the above predictions provide a plausible mechanistic explanation for the increase in ionic conductivity observed in stretched electrolytes [16,17,21]. Indeed, upon stretching a material system with the above characteristics, the polymer chains in both the amorphous and crystalline phases tend to align with the stretching direction [6]; the effective mobility along the stretching direction should thus tend to $m_{\alpha}^{\|}$. For the choice $c_{0 \alpha}^{(\mathrm{cr})} / c_{0 \alpha}^{(a)}=10, m_{\alpha}^{\|} / m_{\alpha}^{\perp}=1,000$, and $\left(m_{\alpha}^{\|}+m_{\alpha}^{\perp}\right) /\left(2 m_{\alpha}^{(a)}\right)=5$, the mobility 
(a)

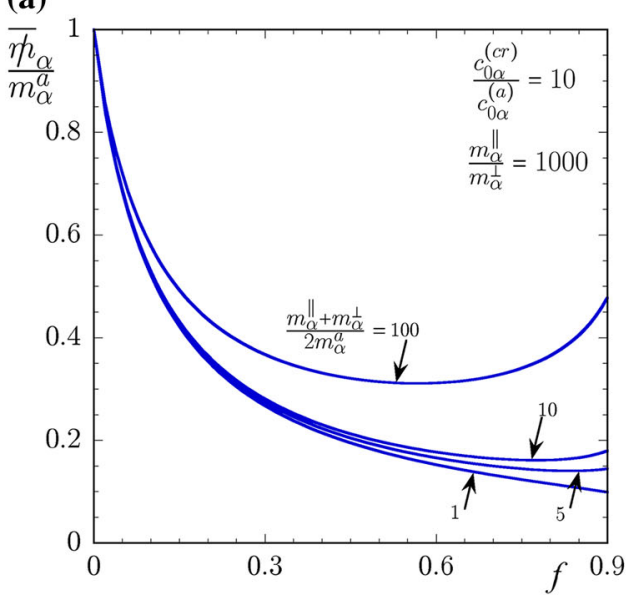

(c)

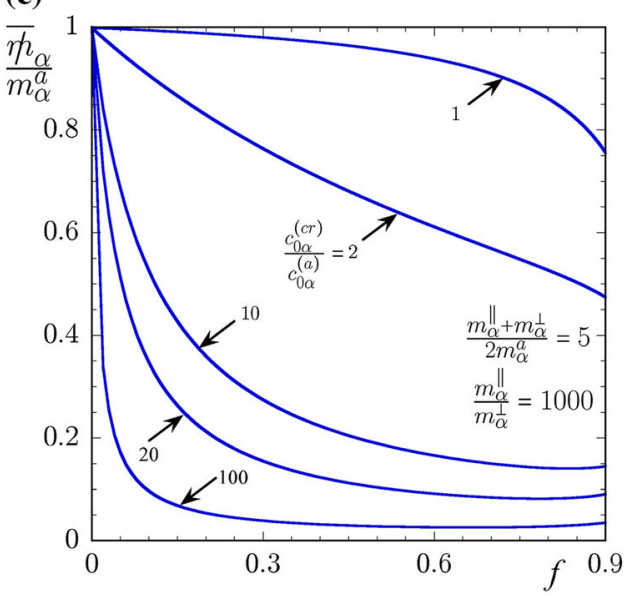

(b)

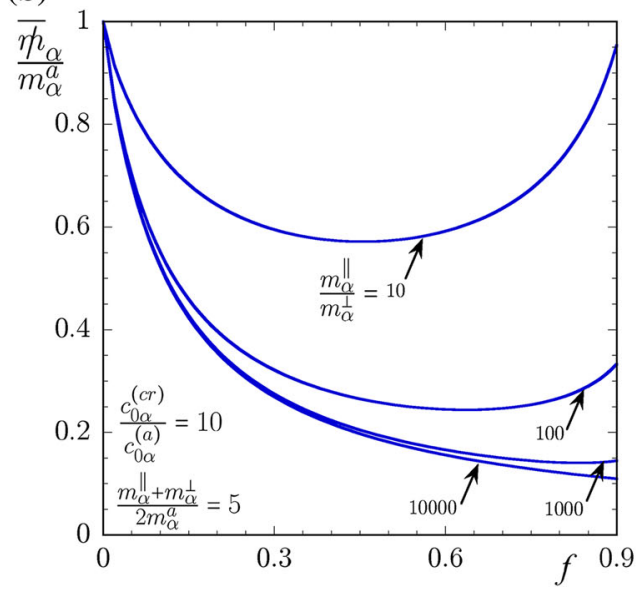

(d)

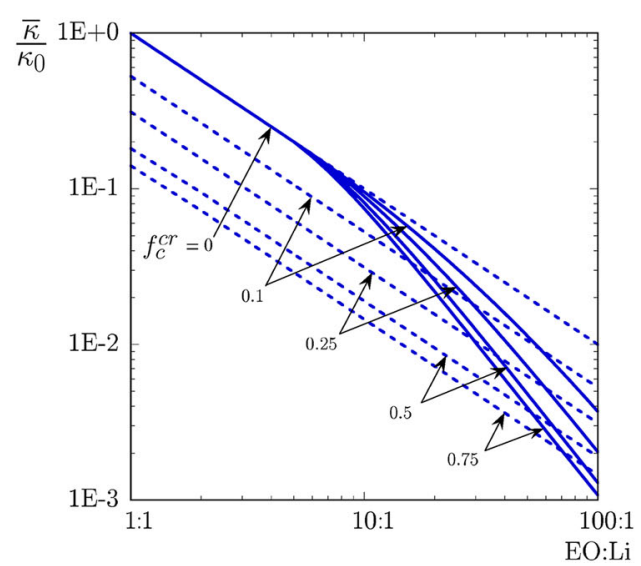

Fig. 2 a-c Effective mobility of species $\alpha$ in a semicrystalline polymer electrolyte without amorphous rings $\left(f_{r}=0\right)$, normalized by the corresponding mobility $m_{\alpha}^{(a)}$ in the amorphous phase, versus crystallinity $f$; plots for: $\mathbf{a} c_{0 \alpha}^{(\text {cr) }} / c_{0 \alpha}^{(a)}=10, m_{\alpha}^{\|} / m_{\alpha}^{\perp}=1,000$, and various mobility contrasts $\left(m_{\alpha}^{\|}+m_{\alpha}^{\perp}\right) /\left(2 m_{\alpha}^{(a)}\right) ; \mathbf{b} c_{0 \alpha}^{(\mathrm{cr})} / c_{0 \alpha}^{(a)}=10,\left(m_{\alpha}^{\|}+m_{\alpha}^{\perp}\right) /\left(2 m_{\alpha}^{(a)}\right)=5$, and various mobility anisotropy ratios $m_{\alpha}^{\|} / m_{\alpha}^{\perp} ; \mathbf{c}\left(m_{\alpha}^{\|}+m_{\alpha}^{\perp}\right) /\left(2 m_{\alpha}^{(a)}\right)=5, m_{\alpha}^{\|} / m_{\alpha}^{\perp}=1,000$, and various reference concentration contrasts $c_{0 \alpha}^{(\mathrm{cr})} / c_{0 \alpha}^{(a)}$. d Effective conductivity of a class of electrolytes with varying microstructure, normalized by a reference conductivity $\kappa_{0}$, as a function of the PEO ether oxygens to lithium ions ratio (EO:Li), for various crystallinity levels of the cylindrites $f_{c}^{\text {cr }}$

$m_{\alpha}^{\|}$can be two orders of magnitude larger than the predicted effective mobility for (unstretched) systems with moderate crystallinity levels. This is approximately the increase reported by [15] in PEO systems doped with LiI.

The predictions provided in part (c) show the role of the reference concentration contrast on the effective mobility for the choice $m_{\alpha}^{\|} / m_{\alpha}^{\perp}=1,000$ and $\left(m_{\alpha}^{\|}+m_{\alpha}^{\perp}\right) /\left(2 m_{\alpha}^{(a)}\right)=5$. It is seen that realistic trends are reproduced provided $c_{0 \alpha}^{(\mathrm{cr})}>2 c_{0 \alpha}^{(a)}$. Thus, the usual assumption $c_{0 \alpha}^{(\mathrm{cr})}=c_{0 \alpha}^{(a)}$, which implies that the ion concentrations are continuous and consequently simplify the mathematical formulation, is at odds not only with the expected inhomogeneity of local ion concentration at equilibrium but also with the observed macroscopic variation of conductivity with crystallinity.

In this connection, it should be pointed out that polymer electrolytes with different effective conductivity always differ in several microstructural and material characteristics, making it difficult to experimentally isolate the effect of individual parameters. For instance, the work of [13] suggests that, as dopant concentration at casting increases, the amorphous phase surrounding the spherulites takes on a larger volume fraction of the electrolyte and that the dopant concentration in the crystalline phase remains constant and only increases in the amorphous phase. By way of example, part (d) shows predictions for the effective conductivity as a function of dopant molar concentration at casting $c$, accounting for such variations in microstructural and 
material parameters. Note that the ratio $\left(c / c_{0}\right)^{-1}$ represents the total ratio of PEO ether oxygens to lithium ions (EO:Li) in the as-cast electrolyte. The predictions correspond to $\bar{c}_{+}=c$ and lithium mobilities such that $m_{+}^{\|} / m_{+}^{\perp}=1,000$ and $\left(m_{+}^{\|}+m_{+}^{\perp}\right) /\left(2 m_{+}^{a}\right)=5$. In turn, the volume fraction of amorphous ring and reference concentration ratio are taken to depend on $c$ as

$$
f_{r}=\gamma_{r}\left(\frac{c}{c_{0}}\right) \text { and } \frac{c_{0+}^{(\mathrm{cr})}}{c_{0+}^{(a)}}=\gamma_{c}\left(\frac{c}{c_{0}}\right)^{-1},
$$

with $\gamma_{r}=1 / \gamma_{c}=5$; these numerical constants follow roughly from the data of [13]. For reference, the figure also includes the predictions for systems with no amorphous rings $\left(f_{r}=0\right)$ and fixed $c_{0+}^{(\mathrm{cr})} / c_{0+}^{(a)}=10$ (dotted lines). The main observation in the context of this figure is that a variation of microstructural and material parameters of the form (67) can increase the variability of the effective conductivity with dopant concentration by an order of magnitude. Note that for $f_{c}^{\text {cr }}=0.75$, the variation is three orders of magnitude, which falls within the range observed by [13]. The break point in these curves corresponds to the EO:Li ratio below which the material is fully amorphous. It should be pointed out, however, that additional microstructural changes are expected to occur which can modify the predicted trends. For instance, dopant molecules may segregate and form second-phase particles at larger concentrations (e.g., [19]). The above theoretical framework can certainly incorporate such features provided sufficient experimental guidance is available.

\subsection{The effect of nonconducting fillers}

In addition to ionic conductivity, the transport numbers

$$
\bar{t}_{+}=\frac{\overline{\eta ্}_{+}}{\overline{\eta ্}_{+}+\overline{\eta ̆}_{-}} \text {and } \bar{t}_{-}=1-\bar{t}_{+}=\frac{\overline{\eta ্}_{-}}{\overline{\bar{h}}_{+}+\overline{\eta ̆}_{-}}
$$

are electrolyte properties of particular relevance to battery performance-see, for instance, [12]. In general, high cation transport numbers $\left(\bar{t}_{+}\right)$seem desirable. The cation transport number in PEO systems doped with $\mathrm{LiClO}_{4}$ is low $(\sim 0.25$ at room temperature) but can increase significantly with the addition of a ceramic filler such as $\mathrm{Al}_{2} \mathrm{O}_{3}$ and $\mathrm{ZrO}_{2}$ - see, for instance, $[8,10,11]$. The above Schulgasser assemblage model can be enriched to assess the role of interphases in this effect.

In this case, the cylindrites are assumed to contain a central inclusion with vanishing mobilities and reference concentrations representing the nonconducting filler, surrounded by an isotropic layer with mobilities $m_{\alpha}^{\text {(int) }}$ and reference concentrations $c_{0 \alpha}^{(\mathrm{int})}$, see Fig. 1. This microgeometry is consistent with the observation that filler particles act as nucleation sites for spherulites during casting and are surrounded by amorphous interphases of finite size (e.g., [24]). The effective mobilities of this assemblage are obtained, once again, following the scheme described in the previous section. The result is

$$
\bar{\eta}_{\alpha}=\eta_{\alpha}^{(a)} \frac{\left(1+f_{c}\right) A_{\alpha}+\left(1-f_{c}\right) \eta_{\alpha}^{(a)}}{\left(1-f_{c}\right) A_{\alpha}+\left(1+f_{c}\right) \eta_{\alpha}^{(a)}},
$$

where

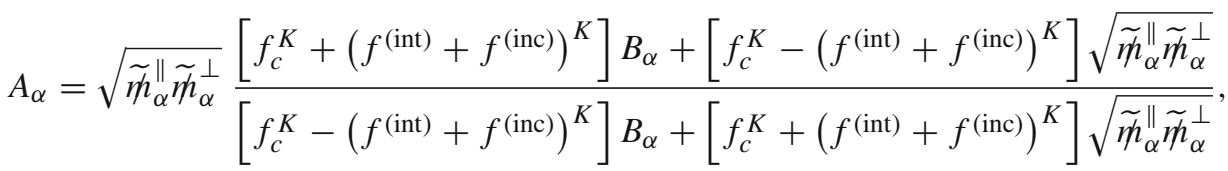

$$
\begin{aligned}
& B_{\alpha}=h_{\alpha}^{(\mathrm{int})} \frac{f^{(\mathrm{int})}}{f^{\text {(int) }}+2 f^{\text {(inc) }}} .
\end{aligned}
$$

In these expressions, $f^{(\text {inc) }}$ and $f^{\text {(int) }}$ denote the volume fractions of inclusions and interfaces, respectively, $f_{c}$ is the volume fraction of the core composed of the inclusion, the interphase, and the cylindrite; the weighted mobilities are given by

$$
\not h_{\alpha}^{(r)}=\frac{c_{0 \alpha}^{(r)}}{f^{(\mathrm{cr})} c_{0 \alpha}^{(\mathrm{cr})}+f^{(a)} c_{0 \alpha}^{(a)}+f^{(\mathrm{int})} c_{0 \alpha}^{(\mathrm{int})}} m_{\alpha}^{(r)}
$$


(a)

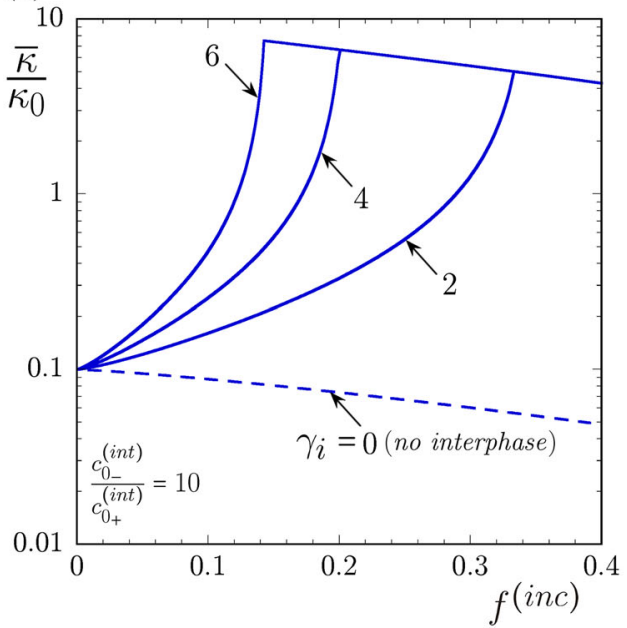

(b)

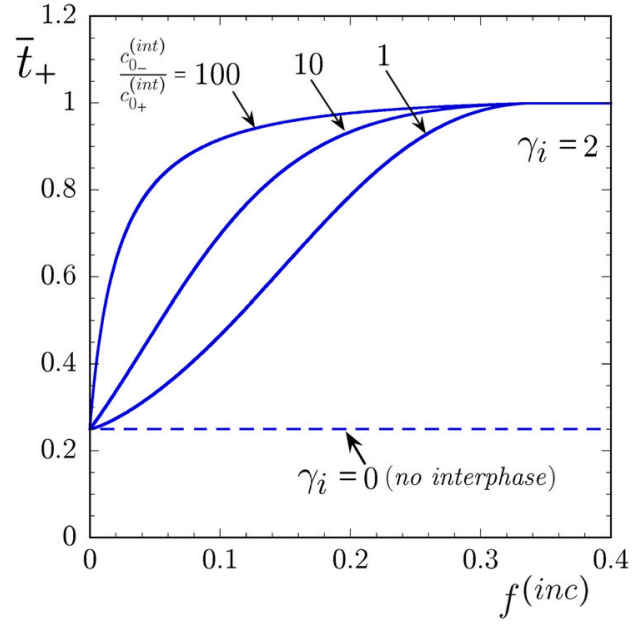

Fig. 3 Transport properties of a composite electrolyte $(\mathrm{EO}: \mathrm{Li}=10)$ as a function of filler content, for various interphase growth rates and reference concentration ratios $c_{0-}^{\text {(int) }} / c_{0+}^{(\text {int) }}$ : a effective conductivity, normalized by the reference conductivity $\kappa_{0}$; b effective cation transport number

( $r=a, \mathrm{cr}$, int), and $K=\sqrt{\widetilde{m}_{\alpha}^{\|} / \widetilde{m}_{\alpha}^{\perp}}$. Details of the derivation are provided in Appendix 2. Under steady state conditions, the effective conductivity is given by (65) with $\overline{\eta ̆}_{+}$now given by (69).

Sample numerical results are reported for the choice $f_{c}^{\mathrm{cr}}=1-f_{c}^{a}=0.25, f_{r}=1-f_{c}=0.6(1-$ $\left.f^{(\text {inc) }}-f^{(\text {int })}\right), c_{0 \alpha}^{(\text {(r) }} / c_{0 \alpha}^{(a)}=1, m_{\alpha}^{\|} / m_{\alpha}^{\perp}=1,000$ and $\left(m_{\alpha}^{\|}+m_{\alpha}^{\perp}\right) /\left(2 m_{\alpha}^{(a)}\right)=5$ for both ionic species, and $m_{-}^{(a)}=3 m_{+}^{(a)}$. Thus, anion mobilities outside the interphase are three times higher than the corresponding cation mobilities, giving a cation transport number in the absence of fillers of $\bar{t}^{+}=1 /(1+3)=0.25$. However, to reproduce an anchoring effect of anions in a good cation-conducting interphase, we set $m_{-}^{(\text {int })}=0.033 m_{-}^{(a)}$ and $m_{+}^{(\text {int })}=100 m_{+}^{(a)}, c_{0+}^{(\text {int })} / c_{0+}^{(a)}=1$ and high ratios $c_{0-}^{(\text {int })} / c_{0-}^{(a)}$ : A high reference concentration $c_{0-}^{(\text {int }) ~ " a t t r a c t s " ~}$ anions to the interphase, while a low mobility $m_{-}^{\text {(int) }}$ precludes them to move away from it. We further take $\bar{c}_{+}=\left(1-f^{(\text {inc) }}\right) c$ with $c / c_{0}=1 / 10$ (i.e., $\left.\mathrm{EO}: \mathrm{Li}=10: 1\right)$ and assume that the interphase size depends linearly on filler size according to

$$
f^{\text {(int) }}=\gamma_{i} f^{\text {(inc) }} \text {. }
$$

Figure 3 shows predictions for the effective conductivity and cation transport number as a function of filler content for various interphase growth rates $\gamma_{i}$ and reference concentration ratios $c_{0-}^{(\text {int })} / c_{0-}^{(a)}$. In the absence of interphases $\left(\gamma_{i}=0\right)$, the cation transport number is independent of filler content and the effective conductivity decreases moderately with increasing filler content as a result of the insulating effect of the inclusions and of the decrease in overall lithium molar density. In the presence of interphases, by contrast, both properties vary strongly with filler content. In the case of the effective conductivity, predictions are seen to increase initially by up to two orders of magnitude and reach a maximum when the interphases percolate-interphase percolation occurs at $f^{\text {(inc) }}=1 /\left(1+\gamma_{i}\right)$ for the choice (73), see part (a). From there on, the model material becomes a two-phase composite made up of an isotropic conducting matrix and nonconducting inclusions; thus, the ionic conductivity decreases monotonically with increasing filler content. These trends are consistent with experimental data from various PEO composites [24], which consistently show a maximum within the range of 0.05-0.3 filler content. Thus, the model suggests that interphase percolation may indeed be one of the mechanisms behind the observed maximum in those material systems.

In the case of the cation transport number, predictions are also seen to increase with filler content up to the point where interphases percolate, see part (b). The larger the anion reference concentration $c_{0-}^{\text {(int) }}$ the steeper the increase. Beyond percolation, $\bar{t}_{+}$remains constant and close to unity. Thus, a combination of high $c_{0-}^{\text {(int) }}$ with low $m_{-}^{\text {(int) }}$ does seem to reproduce an anchoring effect of anions within the interphase region. In particular, 
the choice $c_{0-}^{(\mathrm{int})} / c_{0+}^{(\mathrm{int})}=10$ reproduces an increase of cation transport number with filler content comparable to those reported for PEO composites [10] and other composite polymer electrolytes [25]. This makes plain the importance of allowing for spatial variations of the reference concentration when modeling microstructured solid electrolytes.

\section{Concluding remarks}

In the above model problem, the field equations were homogenized for specific forms of the energy functions $W^{(r)}$ and dissipation potentials $U^{(r)}$, as given by (12)-(13). These forms pertain to linearly polarizable solids containing dilute solutions of ions showing linear transport behavior. However, the homogenization procedure could handle more complicated energy and dissipation functions describing additional phenomena such as nonlinear polarizability and strong ion-ion interactions in nondilute solutions. Such nonlinear analyses are required, for instance, to estimate the influence of microstructural parameters on the dielectric strength and electrochemical stability of a solid electrolyte, properties that are of particular relevance to the design of rechargeable batteries. This possibility is currently being pursued and will be the subject of a separate contribution.

Acknowledgments This work was funded by the Agencia Nacional de Promoción Científica y Tecnológica through grants PRH-2007-15 and PICT-2011-0167. Additional support from the Universidad Nacional de La Plata through grant I-2013-179 is also gratefully acknowledged.

\section{Appendix 1: Multi-scale limits}

We assume the fields $\mu_{\alpha}^{\varepsilon}(\mathbf{x})$ and $\phi^{\varepsilon}(\mathbf{x})$ solving Eqs. (19)-(20) are bounded in $H_{0}^{1}(\Omega)$ for all $\varepsilon$. Then, up to a subsequence, these fields multi-scale converge to some limiting fields $\bar{\mu}_{\alpha}(\mathbf{x})$ and $\bar{\phi}(\mathbf{x})$ in $H_{0}^{1}(\Omega)$, respectively, while their gradients multi-scale converge to some limiting gradient fields $\nabla \bar{\mu}_{\alpha}(\mathbf{x})+\nabla_{y} \mu_{\alpha}^{1}(\mathbf{x}, \mathbf{y})+\nabla_{z} \mu_{\alpha}^{2}(\mathbf{x}, \mathbf{y}, \mathbf{z})$ and $\nabla \bar{\phi}(\mathbf{x})+\nabla_{y} \phi^{1}(\mathbf{x}, \mathbf{y})+\nabla_{z} \phi^{2}(\mathbf{x}, \mathbf{y}, \mathbf{z})$, respectively, with the functions $\mu_{\alpha}^{i}$ and $\phi^{i}(\mathbf{x}, \cdot) \Omega_{\#}$-periodic in the $\mathbf{y}$ and $\mathbf{z}$ arguments—see [3]. The equations for the various limiting functions are obtained next by writing the field Eqs. (19)-(20) in weak form and passing to the limit $\varepsilon \rightarrow 0$.

Diffusion equations. For a given sequence of electric potentials $\phi^{\varepsilon}$, Eq. (19) for the chemical potentials $\mu_{\alpha}^{\varepsilon}$ can be written in weak form as

$$
\int_{\Omega}\left[\nabla \cdot \mathbb{D}_{\alpha}\left(\frac{\mathbf{x}}{\varepsilon}, \frac{\mathbf{x}}{\varepsilon^{2}}, \mu_{\alpha}^{\varepsilon}, \phi^{\varepsilon}\right) \nabla \mu_{\alpha}^{\varepsilon}+h_{\alpha}(\mathbf{x})\right] \varphi(\mathbf{x}) \mathrm{d} V=0 \quad \forall \varphi \in H_{0}^{1}(\Omega) .
$$

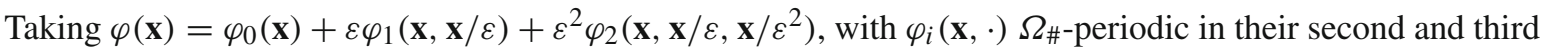
arguments, and integrating by parts, we obtain

$$
\lim _{\varepsilon \rightarrow 0} \int_{\Omega} \nabla \mu_{\alpha}^{\varepsilon} \cdot \mathbb{D}_{\alpha}\left(\frac{\mathbf{x}}{\varepsilon}, \frac{\mathbf{x}}{\varepsilon^{2}}, \mu_{\alpha}^{\varepsilon}, \phi^{\varepsilon}\right)\left[\nabla \varphi_{0}(\mathbf{x})+\nabla_{y} \varphi_{1}(\mathbf{x}, \mathbf{x} / \varepsilon)+\nabla_{z} \varphi_{2}\left(\mathbf{x}, \mathbf{x} / \varepsilon, \mathbf{x} / \varepsilon^{2}\right)\right] \mathrm{d} V=\int_{\Omega} h_{\alpha}(\mathbf{x}) \varphi_{0}(\mathbf{x}) \mathrm{d} V
$$

for all admissible functions $\varphi_{0}, \varphi_{1}$, and $\varphi_{2}$. Based on the work of [7] on quasi-linear operators oscillating at a single length scale and satisfying certain polynomial growth conditions, it is reasonable to expect that the equality

$$
\begin{aligned}
& \lim _{\varepsilon \rightarrow 0} \int_{\Omega} \nabla \mu_{\alpha}^{\varepsilon} \cdot \mathbb{D}_{\alpha}\left(\frac{\mathbf{x}}{\varepsilon}, \frac{\mathbf{x}}{\varepsilon^{2}}, \mu_{\alpha}^{\varepsilon}, \phi^{\varepsilon}\right)\left[\nabla \varphi_{0}(\mathbf{x})+\nabla_{y} \varphi_{1}(\mathbf{x}, \mathbf{x} / \varepsilon)+\nabla_{z} \varphi_{2}\left(\mathbf{x}, \mathbf{x} / \varepsilon, \mathbf{x} / \varepsilon^{2}\right)\right] \mathrm{d} V \\
& \quad=\lim _{\varepsilon \rightarrow 0} \int_{\Omega} \nabla \mu_{\alpha}^{\varepsilon} \cdot \mathbb{D}_{\alpha}\left(\frac{\mathbf{x}}{\varepsilon}, \frac{\mathbf{x}}{\varepsilon^{2}}, \bar{\mu}_{\alpha}, \bar{\phi}\right)\left[\nabla \varphi_{0}(\mathbf{x})+\nabla_{y} \varphi_{1}(\mathbf{x}, \mathbf{x} / \varepsilon)+\nabla_{z} \varphi_{2}\left(\mathbf{x}, \mathbf{x} / \varepsilon, \mathbf{x} / \varepsilon^{2}\right)\right] \mathrm{d} V
\end{aligned}
$$


holds $^{2}$. Granted this assumption, equation (74) and the definition of multi-scale convergence imply that

$$
\begin{aligned}
& \int_{\Omega}\left\langle\left[\nabla \bar{\mu}_{\alpha}(\mathbf{x})+\nabla_{y} \mu_{\alpha}^{1}(\mathbf{x}, \mathbf{y})+\nabla_{z} \mu_{\alpha}^{2}(\mathbf{x}, \mathbf{y}, \mathbf{z})\right] \cdot \mathbb{D}_{\alpha}\left(\mathbf{y}, \mathbf{z}, \bar{\mu}_{\alpha}, \bar{\phi}\right)\left[\nabla \varphi_{0}(\mathbf{x})+\nabla_{y} \varphi_{1}(\mathbf{x}, \mathbf{y})+\nabla_{z} \varphi_{2}(\mathbf{x}, \mathbf{y}, \mathbf{z})\right]\right\rangle_{y, z} \mathrm{~d} V \\
& \quad=\int_{\Omega} h_{\alpha}(\mathbf{x}) \varphi_{0}(\mathbf{x}) \mathrm{d} V
\end{aligned}
$$

for all admissible functions $\varphi_{0}, \varphi_{1}$, and $\varphi_{2}$. Finally, variations with respect to $\varphi_{0}, \varphi_{1}$, and $\varphi_{2}$ deliver the field Eqs. (25) and (29).

Gauss' equation. Noting that the function $g$ is the derivative of a convex function $f$, it is easy to show that, for given fields $\mu_{\alpha}^{\varepsilon}$, the electric potential $\phi^{\varepsilon}$ satisfying (20) solves the minimization problem

$$
\bar{I}^{\varepsilon}=\min _{\phi^{\varepsilon} \in H_{0}^{1}(\Omega)} I^{\varepsilon}\left[\phi^{\varepsilon}\right]=\min _{\phi^{\varepsilon} \in H_{0}^{1}(\Omega)} \int\left[\frac{1}{2} \nabla \phi^{\varepsilon} \cdot \boldsymbol{\epsilon}\left(\frac{\mathbf{x}}{\varepsilon}, \frac{\mathbf{x}}{\varepsilon^{2}}\right) \nabla \phi^{\varepsilon}+\sum_{\alpha=1}^{A} c_{0 \alpha}\left(\frac{\mathbf{x}}{\varepsilon}, \frac{\mathbf{x}}{\varepsilon^{2}}\right) f\left(\mu_{\alpha}^{\varepsilon}-F z_{\alpha} \phi^{\varepsilon}\right)\right] \mathrm{d} V .
$$

The functional $I^{\varepsilon}\left[\phi^{\varepsilon}\right]$ can be bounded from above and below as follows. On the one hand, the convexity of the integrand of (78) in $\nabla \phi^{\varepsilon}$ and $\phi^{\varepsilon}$ implies that

$I^{\varepsilon}\left[\phi^{\varepsilon}\right] \geq \int_{\Omega}\left[\frac{1}{2} \mathbf{e} \cdot \boldsymbol{\epsilon}\left(\frac{\mathbf{x}}{\varepsilon}, \frac{\mathbf{x}}{\varepsilon^{2}}\right) \mathbf{e}+\boldsymbol{\epsilon}\left(\frac{\mathbf{x}}{\varepsilon}, \frac{\mathbf{x}}{\varepsilon^{2}}\right) \mathbf{e} \cdot\left(\nabla \phi^{\varepsilon}-\mathbf{e}\right)+\sum_{\alpha=1}^{A} c_{0 \alpha}\left(\frac{\mathbf{x}}{\varepsilon}, \frac{\mathbf{x}}{\varepsilon^{2}}\right)\left[f\left(s_{\alpha}\right)+f^{\prime}\left(s_{\alpha}\right)\left(\mu_{\alpha}^{\varepsilon}-F z_{\alpha} \phi^{\varepsilon}-s_{\alpha}\right)\right]\right] \mathrm{d} V$

for every $\varepsilon$ and any fields $\mathbf{e}(\mathbf{x})$ and $s_{\alpha}(\mathbf{x})$. In particular, take

$$
\mathbf{e}(\mathbf{x})=\nabla \bar{\phi}(\mathbf{x})+\nabla_{y} \phi^{1}(\mathbf{x}, \mathbf{x} / \varepsilon)+\nabla_{z} \phi^{2}\left(\mathbf{x}, \mathbf{x} / \varepsilon, \mathbf{x} / \varepsilon^{2}\right) \text { and } s_{\alpha}(\mathbf{x})=\bar{\mu}_{\alpha}(\mathbf{x})-F z_{\alpha} \bar{\phi}(\mathbf{x}) .
$$

By definition of multi-scale convergence, we obtain the lower bound

$$
\lim _{\varepsilon \rightarrow 0} I^{\varepsilon}\left[\phi^{\varepsilon}\right] \geq I\left[\bar{\phi}, \phi^{1}, \phi^{2}\right]
$$

where

$$
\begin{aligned}
I\left[\bar{\phi}, \phi^{1}, \phi^{2}\right]= & \int_{\Omega}\left\langle_{\frac{1}{2}}\left[\nabla \bar{\phi}(\mathbf{x})+\nabla_{y} \phi^{1}(\mathbf{x}, \mathbf{y})+\nabla_{z} \phi^{2}(\mathbf{x}, \mathbf{y}, \mathbf{z})\right] \cdot \boldsymbol{\epsilon}(\mathbf{y}, \mathbf{z})\left[\nabla \bar{\phi}(\mathbf{x})+\nabla_{y} \phi^{1}(\mathbf{x}, \mathbf{y})+\nabla_{z} \phi^{2}(\mathbf{x}, \mathbf{y}, \mathbf{z})\right]\right. \\
& \left.+\sum_{\alpha=1}^{A} c_{0 \alpha}(\mathbf{y}, \mathbf{z}) f\left(\bar{\mu}_{\alpha}(\mathbf{x})-F z_{\alpha} \bar{\phi}(\mathbf{x})\right)\right\rangle_{y, z} \mathrm{~d} V .
\end{aligned}
$$

On the other hand, evaluating the functional $I^{\varepsilon}$ in (78) at the sequence of potential fields

$$
\varphi^{\varepsilon}(\mathbf{x})=\bar{\phi}(\mathbf{x})+\varepsilon \phi^{1}(\mathbf{x}, \mathbf{x} / \varepsilon)+\varepsilon^{2} \phi^{2}\left(\mathbf{x}, \mathbf{x} / \varepsilon, \mathbf{x} / \varepsilon^{2}\right)+\frac{1}{F z_{\alpha}}\left[\mu_{\alpha}^{\varepsilon}(\mathbf{x})-\bar{\mu}_{\alpha}(\mathbf{x})-\varepsilon \mu_{\alpha}^{1}(\mathbf{x}, \mathbf{x} / \varepsilon)-\varepsilon^{2} \mu_{\alpha}^{2}\left(\mathbf{x}, \mathbf{x} / \varepsilon, \mathbf{x} / \varepsilon^{2}\right)\right],
$$

and invoking the definition of two-scale convergence, we obtain the upper bound

$$
\lim _{\varepsilon \rightarrow 0} I^{\varepsilon}\left[\phi^{\varepsilon}\right] \leq \lim _{\varepsilon \rightarrow 0} I^{\varepsilon}\left[\varphi^{\varepsilon}\right]=I\left[\bar{\phi}, \phi^{1}, \phi^{2}\right]
$$

since $\phi^{\varepsilon}$ is the minimizer of $I^{\varepsilon}$. Noting that the lower bound (81) and the upper bound (84) coincide, we conclude that

$$
\lim _{\varepsilon \rightarrow 0} I^{\varepsilon}\left[\phi^{\varepsilon}\right]=I\left[\bar{\phi}, \phi^{1}, \phi^{2}\right] .
$$

Thus, following the arguments of [2], the multi-scale fields $\bar{\phi}, \phi^{1}$, and $\phi^{2}$ should minimize $I\left[\bar{\phi}, \phi^{1}, \phi^{2}\right]$. It is easy to show that the minimization conditions imply the field Eqs. (26) and (30).

\footnotetext{
2 A rigorous proof for the growth conditions (15) is not available.
} 


\section{Appendix 2: Effective properties of Schulgasser assemblages}

Consider a two-dimensional Schulgasser assemblage composed of homothetic cylinders with radial and tangential local mobilities $m^{\|}(\rho)$ and $m^{\perp}(\rho)$, which can vary arbitrarily with the radial coordinate $\rho \in[0,1]$ within the cylinders. The extreme values $\rho=0$ and $\rho=1$ correspond to the center and periphery of a cylinder, respectively. Schulgasser (1983) has shown ${ }^{3}$ that the effective mobility $\bar{m}$ of the assemblage is given by-see also [20]-

$$
\bar{m}=h(1),
$$

where the function $h(\rho) \in C^{0}[0,1]$ is solution to the Ricatti equation

$$
\rho h^{\prime}(\rho)+\frac{[h(\rho)]^{2}}{m^{\perp}(\rho)}=m^{\|}(\rho) .
$$

Anisotropic core with isotropic ring. Consider the case of a cylinder with an anisotropic central core of radius $\rho_{c}$ with mobilities $m_{c}^{\|, \perp}$ and an isotropic external ring with mobility $m_{r}$. Then,

$$
m^{\|, \perp}(\rho)= \begin{cases}m_{c}^{\|, \perp} & 0 \leq \rho \leq \rho_{c} \\ m_{r} & \rho_{c}<\rho \leq 1\end{cases}
$$

Integrating (87) piecewise and enforcing continuity at $\rho=\rho_{c}$, we obtain

$$
h(\rho)= \begin{cases}\sqrt{m_{c}^{\|} m_{c}^{\perp}} & 0 \leq \rho \leq \rho_{c}, \\ m_{r} \frac{\left(\sqrt{m_{c}^{\|} m_{c}^{\perp}}+m_{r}\right)\left(\rho / \rho_{c}\right)^{2}+\left(\sqrt{m_{c}^{\|} m_{c}^{\perp}}-m_{r}\right)}{\left(\sqrt{m_{c}^{\|} m_{c}^{\perp}}+m_{r}\right)\left(\rho / \rho_{c}\right)^{2}-\left(\sqrt{m_{c}^{\|} m_{c}^{\perp}}-m_{r}\right)} & \rho_{c}<\rho \leq 1 .\end{cases}
$$

At $\rho=1$, we have that $\left(\rho / \rho_{c}\right)^{2}=1 / f_{c}$ where $f_{c}$ is the volume fraction of the core. Thus, after routine algebra, we finally obtain

$$
\bar{m}=h(1)=m_{r} \frac{\left(1+f_{c}\right) \sqrt{m_{c}^{\|} m_{c}^{\perp}}+\left(1-f_{c}\right) m_{r}}{\left(1-f_{c}\right) \sqrt{m_{c}^{\|} m_{c}^{\perp}}+\left(1+f_{c}\right) m_{r}} .
$$

Composite core with isotropic ring. Now consider the case of a cylinder composed of an isotropic central inclusion with an isotropic concentric interface within the core. Then,

$$
m^{\|, \perp}(\rho)= \begin{cases}m_{\text {inc }} & 0 \leq \rho<\rho_{\text {inc }} \\ m_{\text {int }} & \rho_{\text {inc }} \leq \rho<\rho_{\text {int }} \\ m_{c}^{\|, \perp} & \rho_{\text {int }} \leq \rho<\rho_{c} \\ m_{r} & \rho_{c} \leq \rho \leq 1\end{cases}
$$

Integrating (87) piecewise and enforcing continuity of the solution, we obtain

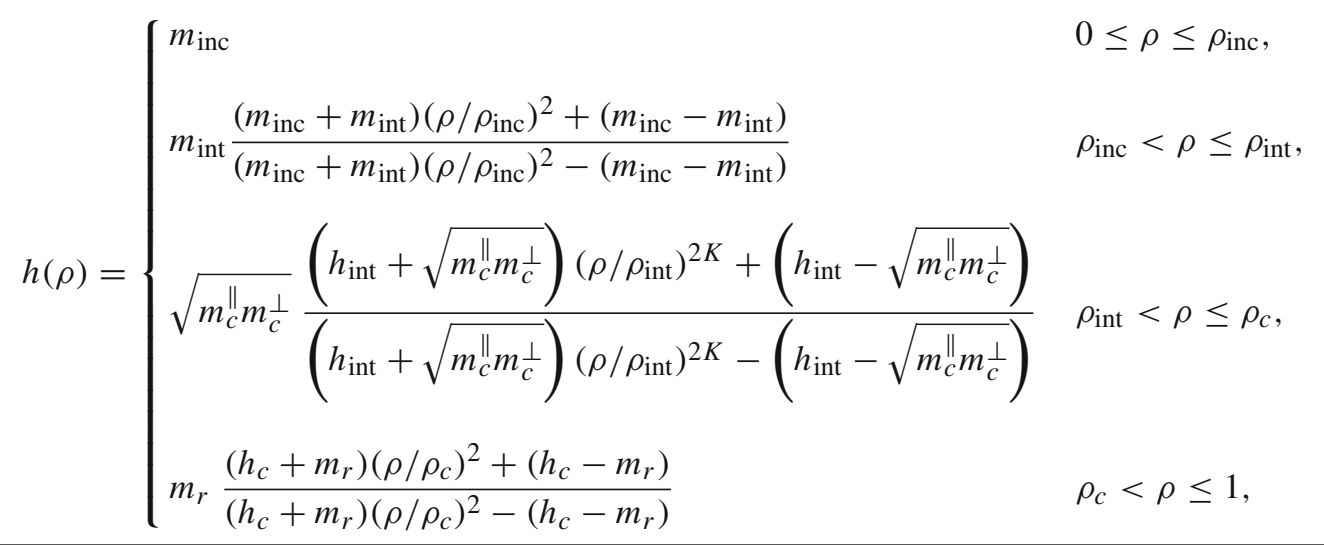

\footnotetext{
${ }^{3}$ Schulgasser (1983) considered the mathematically analogous problem of thermal conductivity.
} 
where $K=\sqrt{m_{c}^{\|} / m_{c}^{\perp}}$, and

$$
\begin{aligned}
h_{\mathrm{int}} & =h\left(\rho_{\mathrm{int}}\right)=m_{\mathrm{int}} \frac{f_{\mathrm{int}} m_{\mathrm{int}}+\left(f_{\mathrm{int}}+2 f_{\mathrm{inc}}\right) m_{\mathrm{inc}}}{\left(f_{\mathrm{int}}+2 f_{\mathrm{inc}}\right) m_{\mathrm{int}}+f_{\mathrm{int}} m_{\mathrm{inc}}}, \\
h_{c} & =h\left(\rho_{c}\right)=\sqrt{m_{c}^{\|} m_{c}^{\perp}} \frac{\left[f_{c}^{K}+\left(f_{\mathrm{int}}+f_{\mathrm{inc}}\right)^{K}\right] h_{\mathrm{int}}+\left[f_{c}^{K}-\left(f_{\mathrm{int}}+f_{\mathrm{inc}}\right)^{K}\right] \sqrt{m_{c}^{\|} m_{c}^{\perp}}}{\left[f_{c}^{K}-\left(f_{\mathrm{int}}+f_{\mathrm{inc}}\right)^{K}\right] h_{\mathrm{int}}+\left[f_{c}^{K}+\left(f_{\mathrm{int}}+f_{\mathrm{inc}}\right)^{K}\right] \sqrt{m_{c}^{\|} m_{c}^{\perp}}} .
\end{aligned}
$$

Here, $f_{c}, f_{\text {int }}$, and $f_{\text {inc }}$ denote the volume fractions of the core, the interface, and the inclusion, respectively. Thus, at $\rho=1$, we have that $\left(\rho / \rho_{c}\right)^{2}=1 / f_{c}$ and so

$$
\bar{m}=h(1)=m_{r} \frac{\left(1+f_{c}\right) h_{c}+\left(1-f_{c}\right) m_{r}}{\left(1-f_{c}\right) h_{c}+\left(1+f_{c}\right) m_{r}} .
$$

\section{References}

1. Agoras, M., Ponte Castañeda, P.: Homogenization estimates for multi-scale nonlinear composites. Eur. J. Mech. A/Solids 30, 828-843 (2011)

2. Allaire, G.: Homogenization and two-scale convergence. S.I.A.M. J. Math. Anal. 23, 1482-1518 (1992)

3. Allaire, G., Briane, M.: Multiscale convergence and reiterated homogenization. Proc. R. Soc. Edin. 126A, $297-342$ (1996)

4. Berthier, C., Gorecki, W., Minier, M., Armand, M.B., Chabagno, J.M., Rigaud, P.: Microscopic investigation of ionic conductivity in alkali metal salts-poly(ethylene oxide) adducts. Solid State Ion. 11, 91-95 (1983)

5. Bourbatache, K., Millet, O., Aït-Mohtar, A., Amiri, O.: Chloride transfer in cement-based materials. Part 1. Theoretical basis and modelling. Int. J. Numer. Anal. Meth. Geomech. 37, 1614-1627 (2012)

6. Burba, C.M., Woods, L., Millar, S.Y., Pallie, J.: Polymer chain organization in tensile-stretched poly(ethylene oxide)-based polymer electrolytes. Electrochim. Acta 57, 165-171 (2011)

7. Casado-Díaz, J., Gayte, I.: The two-scale convergence method applied to generalized Besicovitch spaces. Proc. R. Soc. Lond. A 458, 2925-2946 (2002)

8. Ciocek, M., Sannier, L., Siekierski, M., Golodnitsky, D., Peled, E., Scrosati, B., Glowinkowski, S., Wieczorek, W.: Ion transport phenomena in polymeric electrolytes. Electrochim. Acta 53, 1409-1416 (2007)

9. Coleman, R.D., Noll, W.: The thermodynamics of elastic materials with heat conduction and viscosity. Arch. Ration. Mech. Anal. 13, 167-178 (1963)

10. Croce, F., Persi, L., Scrosati, B., Serraino-Fiory, F., Plichta, E., Hendrickson, M.A.: Role of the ceramic fillers in enhancing the transport properties of composite polymer electrolytes. Electrochim. Acta 46, 2457-2461 (2001)

11. Croce, F., Sachetti, S.L., Scrosati, B.: Advanced, lithium batteries based on high-performance composite polymer electrolytes. J. Power Sour. 162, 685-689 (2006)

12. Doyle, M., Fuller, T.F., Newman, J.: The importance of the lithium ion transference number in lithium/polymer cells. Electrochim. Acta 39, 2073-2081 (1994)

13. Fullerton-Shirey, S.K., Maranas, J.K.: Effect of $\mathrm{LiClO}_{4}$ on the structure and mobility of PEO-based solid polymer electrolytes. Macromolecules 42, 2142-2156 (2009)

14. Funke, K.: Solid State Ionics: from Michael Faraday to green energy the European dimension. Sci. Technol. Adv. Mater. 14, 043502 (2013)

15. Gitelman, L., Israeli, M., Averbuch, A., Nathan, M., Schuss, Z., Golodnitsky, D.: Polymer geometry and Li ${ }^{+}$conduction in poly(ethylene oxide). J. Comp. Phys. 227, 8437-8447 (2008)

16. Golodnitsky, D., Peled, E.: Stretching-induced conductivity enhancement of LiI-(PEO)-polymer electrolyte. Electrochim. Acta 45, 1431-1436 (2000)

17. Golodnitsky, D., Livshits, E., Ulus, A., Barkay, Z., Lapides, I., Peled, E., Chung, S.H., Greenbaum, S.: Fast ion transport phenomena in oriented semicrystalline LiI-P(EO) $)_{n}$-based polymer electrolytes. J. Phys. Chem. A 105, 10098-10106 (2001)

18. Gurtin, M.E., Fried, E., Anand, L.: The Mechanics and Thermodynamics of Continua. Cambridge University Press, Cambridge (2010)

19. Marzantowicz, M., Krok, F., Dygas, J.R., Florjańczyk, Z., Zygadlo-Monikowska, E.: The influence of phase segregation on properties of semicrystalline PEO:LiTFSI electrolytes. Solid State Ion. 179, 1670-1678 (2008)

20. Milton, G.W.: The Theory of Composites. Cambridge University Press, Cambridge (2002)

21. Minami, T., Tatsumisago, M., Wakihara, M., Iwakura, C., Kohjiya, S., Tanaka, I.: Solid state ionics for batteries. Springer, Berlin (2005)

22. Robitaille, C.D., Fauteux, D.: Phase diagrams and conductivity characterization of some PEO-LiX electrolytes. J. Electrochem. Soc. 133, 315-325 (1986)

23. Suquet, P.: Elements of homogenization for inelastic solid mechanics. In: Sanchez-Palencia, E., Zaoui, A. (eds.) Homogenization techniques for composite media. Lecture Notes in Physics 272. Springer, Berlin, pp. 193-278 (1987)

24. Siekierski, M., Wieczorek, W., Nadara, K.: Mesoscale models of conductivity in polymeric electrolytes-A comparative study. Electrochim. Acta 53, 1556-1567 (2007)

25. Stephan, A.M., Nahm, K.S.: Review on composite polymer electrolytes for lithium batteries. Polymer 47, 5952-5964 (2006)

26. Xiao, Y., Bhattacharya, K.: A continuum theory of deformable, semiconducting ferroelectrics. Arch. Rational Mech. Anal. 189, 59-95 (2008) 